\title{
General and Specific Approaches to Media Parenting: A Systematic Review of Current Measures, Associations with Screen-Viewing, and Measurement Implications
}

\author{
Russell Jago, PhD, Mark J. Edwards, PhD, Carly R. Urbanski, MSc, and Simon J. Sebire, PhD
}

\section{Abstract}

Background: Parent-focused interventions may help to reduce youth screen-viewing (SV). This review synthesized current information on the links between parenting styles, parenting practices, and youth SV with a focus on measurement.

Methods: A systematic review of electronic databases was conducted.

Results: In all, 29 of 1189 studies met the inclusion criteria. Parenting practices were divided into rule and nonrule-based practices. Seven rules: (1) Limits on total time ( $n$ studies $=23)$; (2) limits on time of day $(n=7)$; (3) content restriction $(n=11)$; $(4)$ mealtime rules $(n=2)$; (5) parental supervision $(n=3)$; (6) contingent screentime $(n=3)$; and (7) no-TV policy $(n=1)$ were reported. Two nonrule-based practices were reported: Co-viewing $(n=6)$ and encouragement to view $(n=2)$. Three studies $(10.3 \%)$ provided information on parenting styles. Only 12 studies (41.4\%) provided information on the reliability/validity of the outcome measure, 15 $(51.7 \%)$ studies provided information on the reliability/validity of the parenting measure, and $6(20.7 \%)$ provided information on the reliability/validity of both outcome and exposure measures.

Conclusions: There is mixed evidence that parenting styles and media-related parenting practices are associated with youth SV. The assessment of parental influence of youth media use is hampered by the diversity of measures that have been used. There is a need for new measures that assess a range of media parenting practices that are relevant to multiple forms of SV.

\section{Introduction}

A dult screen-viewing (SV) time (TV viewing, computer game time, and internet use) has been associated with higher levels of adult obesity, type 2 diabetes, and all-cause mortality. ${ }^{1,2}$ Among youth, SV has been associated with an increased risk of obesity, elevated levels of cardiovascular risk factors, and poor mental wellbeing. ${ }^{3-9}$ Recent evidence suggests that SV patterns track from childhood to adulthood, indicating that reducing youth SV is likely to be important for current and future disease reduction. ${ }^{10}$ The American Academy of Pediatrics (AAP) recommends that children's total media time should be limited to 1-2 hours of quality programming per day. ${ }^{11}$ Data from the National Health and Nutrition Examination Survey (NHANES) indicated that $33 \%$ of youth exceeded the upper threshold of the AAP TV viewing guidelines. ${ }^{12} \mathrm{~A}$ number of national bodies have identified reducing youth $\mathrm{SV}$ as a key public health objective. ${ }^{13,14}$

The mediating variable model suggests that behavior change is facilitated by manipulation of the factors that are associated with SV. ${ }^{15}$ As such, identifying potential intermediate or "mediating" variables and how they may be associated with $\mathrm{SV}$ is a critical element of designing behavior change interventions. A number of studies have shown that parent and child SV patterns are related, with higher parental SV associated with higher child SV, ${ }^{16,17}$ but it is not clear what factors contribute to these associations. To change behavior, more information is needed about how parental influence is manifested. These influences are likely to fall into one of two categoriesparenting style and/or parenting practices including co-viewing. Co-viewing refers to children and parents engaging in SV together. ${ }^{17}$ Parenting styles describe how a 
parent communicates with his/her child. ${ }^{18}$ Four parenting styles have been defined: Authoritarian (demand obedience), authoritative (use reasoning), permissive (acquiesce to child's demands), and uninvolved. ${ }^{18,19}$ Different parenting styles affect the behavior of children. For example, authoritative parenting has been associated with greater consumption of fruit and vegetables amongst youth $^{20,21}$ and permissive parenting styles have been associated with higher levels of physical activity (PA) amongst youth, in the United Kingdom ${ }^{22}$ and the United States. $^{23}$ This suggests that parenting styles may affect energy-balance behaviors differently.

Parenting practices describe context-specific behaviors, such as what a parent does to reduce child SV. ${ }^{24,25}$ A range of different parenting practices including limiting SV time, only allowing screentime at certain times of day, and content restriction have been studied. It is not clear, however, what the patterns of associations between parenting practices, parenting styles, and youth SV are. This information would be helpful in identifying variables to target in future interventions.

There is currently no synthesis of information on what parenting styles and practices have been assessed in relation to youth SV and the patterns of evidence linking particular practices to SV behaviors. Equally, to the best of our knowledge, there has been no attempt to integrate current evidence on the validity and reliability of the parenting styles and practice measures. Without such knowledge, it is difficult to interpret the associations between parenting practices/styles and SV. Therefore, information is needed on the associations that have been examined, the validity and reliability of the measures used to examine those associations, and the current gaps in the measurement literature that require further work. To address these issues, a systematic review was conducted. The review was designed to answer the following research questions:

1. What parenting style and practice variables have been examined in relation to youth SV?

2. What is the evidence for reliability/validity of the parenting style and practice measures that have been used?

3. What are the associations between parenting styles/ practices and youth SV?

4. Are there potentially important parenting styles/practices that have not been assessed?

5. How can the findings from the review inform the development of the next generation of SV parenting measures?

\section{Methods}

A systematic search of seven databases (Cumulative Index to Nursing and Allied Health Literature (CINAHL), EMBASE, International Bibliography of the Social Sciences (IBSS), MEDLINE, PsycINFO, Sociological Abstracts, and Web of Science) was conducted in January, 2012, in accordance with the Preferred Reporting Items for Systematic Reviews and Meta Analyses (PRISMA) guidelines. ${ }^{26}$ A combination of keywords was used, including "child", "adolescent", "parent", "style", "practices", "mediation", "television", "video games", and "computer". (Because there were semantic differences between search engines' definitions of key words, alternatives were used, e.g., "youth" rather than "adolescent", "discipline" opposed to "practice"). After initial screening, reference lists were manually examined to ensure the literature was exhaustively searched.

\section{Inclusion and Exclusion Criteria}

All peer-reviewed studies examining the relationship between parenting practices and/or parenting styles and child or adolescent SV were included within the review. There was no restriction on the publication date of the articles. Searches were conducted in English. Studies were excluded if they: (1) Did not examine either parenting practices or parenting styles in relation to SV; or (2) examined only a narrowly defined element of SV (e.g., viewing of particular content or visits to certain Internet sites). Measures assessing the presence of TV sets in children's bedrooms or eating meals while watching TV were not considered to be parenting practices because the absence of a TV set in a child's bedroom, or whether a child eats while SV, does not necessarily indicate the presence of a rule.

\section{Screening and Data Abstraction}

Duplicate articles were removed prior to screening. Screening and data abstraction were undertaken by two reviewers. Titles and abstracts were reviewed to identify potential articles to include. Full texts of identified papers were screened to identify whether the article should be included in the review. Data from included articles were then independently entered into a purpose-designed data abstraction form. The form contained basic details on the study (sample details, research methods, reliability, and validity of measures used), information on the SV measurement, measure of parenting style and/or practice, and a summary of relevant findings. The type of parenting practice assessed was categorized as either rule or nonrulebased parenting practices. The rule based practices were subdivided into: (1) Limits on total time; (2) limits on time of day; (3) content restriction; (4) rules for no viewing during mealtimes; (5) rules for only allowing viewing when supervised by a parent; (6) contingent screentime in which SV can only occur when other tasks, such as homework or exercise, have been completed; and (7) a noTV policy. There were two nonrule-based practices: (1) Co-viewing (TV/video games), whereby the child had to engage in the behavior at the same time as the parent and (2) parental encouragement to screen view at a particular time of day, and/or encouragement to view particular content. Discussions were held regarding any reporting inconsistencies and a final categorization of each study was agreed. Information on study design, measures (including validity and reliability), analyses, and results were then 
abstracted. Data abstraction forms were transferred into a purpose-designed database and cross-checked against the original forms to minimize any possibility of errors.

\section{Results}

Results are presented in four sections: (1) Summary of studies included in review; (2) tools used to assess parenting styles/practices; (3) quality of measurement tools; and (4) associations between parenting styles/practices and SV.

\section{Summary of the Studies in the Review}

The bibliographic database search yielded 1189 studies, of which 29 articles that were published between 1982 and 2011 satisfied our inclusion and exclusion criteria and were thus included in the final review. ${ }^{27-55}$ A flow chart presenting how the review process was aligned to the PRISMA guidelines including the selection of studies that met the inclusion criteria is presented in Figure 1.

A summary of the studies ${ }^{27-55}$ that were included in the review, including the country, participants, the SV measure assessed, and whether parenting styles or practices were measured, is presented in Table 1. Studies were based overwhelmingly upon data from the United States $(n=18) .^{27,29,31,32,34,37-41,44,45,47,48,51,52,54,55}$ The table indicates that previous work has included children and adolescents with the majority of the studies having samples that were largely white. ${ }^{27-30,32-38,40-55}$ Twenty-seven studies ${ }^{27-44,46-53,55}$ have assessed parenting practices, from which one study has assessed both parenting styles and practices. $^{43}$ Two studies assessed parenting styles alone ${ }^{45,54}$ In terms of participants, 14 studies focused on children, ${ }^{27,28,32,33,35,37,41,43,47,48,50,52-54}$ eight on adolescents, ${ }^{29,31,39,42,45,46,49,55}$ and seven on children and adolescents. ${ }^{30,34,36,38,40,44,51}$ It is noticeable that TV viewing is assessed in all but one of the studies, ${ }^{55}$ with $12^{32,34,35,36,38,39,42,43,46,48,52,54}$ focusing on just TV viewing and 16 focusing on TV viewing and at least one other behavior. ${ }^{27-31,33,37,40,41,44,45,47,49-51,53}$ The one study that did not consider TV viewing focused solely on video game usage. $^{55}$

\section{Measurement Tools}

Parenting styles. A summary of the parenting style measures are presented in Table 2. Of the three studies that assessed parenting styles, one was based on child report, ${ }^{43}$ and two on parent report. ${ }^{45,54}$ The studies made reference to three external published sources. ${ }^{56-58}$

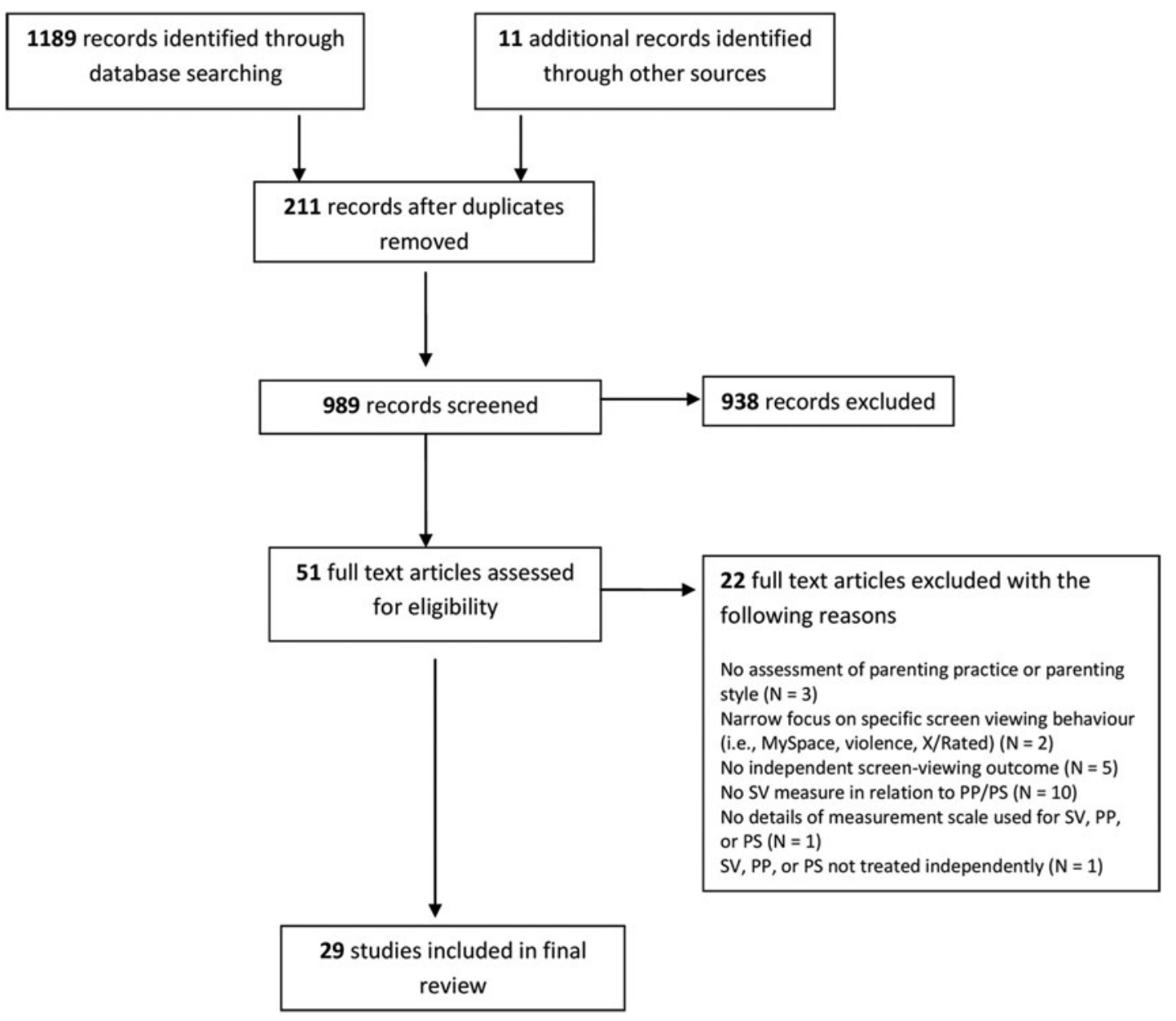

Figure 1. PRISMA flow diagram. 


\begin{tabular}{|c|c|c|c|c|c|c|}
\hline ID & Reference & Country & $\begin{array}{c}\text { Sample details and } \\
\text { child/adolescent demographics }\end{array}$ & Screen viewing measurement & PS & PP \\
\hline I & $\begin{array}{l}\text { Barr et al. } \\
(2010)^{27}\end{array}$ & USA & $\begin{array}{l}\text { - } 308 \text { parents (of infants aged } 6,9,12,15 \text {, } \\
\text { or } 18 \text { months) } \\
\text { - } 167(54.2 \%) \text { male, } 14 \mathrm{I}(45.8 \%) \text { female } \\
\text { - } 71.4 \% \text { white }\end{array}$ & $\begin{array}{l}\text { - TV/DVD/video } \\
\text { - Parent-reported 24-hour } \\
\text { TV/video diary of child's behavior }\end{array}$ & & $\mathbf{x}$ \\
\hline 2 & $\begin{array}{l}\text { He et al. } \\
(2010)^{28}\end{array}$ & Canada & $\begin{array}{l}\text { - } 508 \text { parent-child dyads. } \\
\text { - Grade } 5 \text { \& } 6 \text { children } \\
\text { - } 248(48.8 \%) \text { male, } 260 \text { (5I.2\%) female } \\
\text { - } 77 \% \text { White }\end{array}$ & $\begin{array}{l}\text { - TV, computer, video games } \\
\text { - Child reported: recall of SV on each } \\
\text { day of previous week. }\end{array}$ & & $\mathbf{x}$ \\
\hline 3 & $\begin{array}{l}\text { Lin and Atkin. } \\
(1989)^{29}\end{array}$ & USA & $\begin{array}{l}\text { - } 427 \text { adolescents } \\
\text { - Age range }=I \mid \text { to I3 years }(n=206) \text {, Age } \\
\text { range }=15 \text { to } 17 \text { years }(n=22 I) \\
\text { - } 223(52.2 \%) \text { male, } 203(47.5 \%) \text { female, I } \\
\text { nonreport }\end{array}$ & $\begin{array}{l}\text { - TV and VCR } \\
\text { - Child-reported viewing referred to } \\
\text { hours/day child viewed TV/VCR. }\end{array}$ & & $x$ \\
\hline 4 & $\begin{array}{l}\text { Patriarca et al. } \\
(2009)^{30}\end{array}$ & Italy & $\begin{array}{l}\text { - } 987 \text { children and adolescents } \\
\text { - } M \text {-age }=13.7, S D=1.4 \text {, age range }=1 \mathrm{I}-16 \\
\text { years } \\
\text { - } 5 \mathrm{II}(5 \mathrm{I} .8 \%) \text { male, } 476(48.2 \%) \text { female. }\end{array}$ & $\begin{array}{l}\text { - TV, Video games } \\
\text { - Child-reported questionnaire }\end{array}$ & & $x$ \\
\hline 5 & $\begin{array}{l}\text { Ramirez et al. } \\
(201 \mathrm{I})^{31}\end{array}$ & USA & $\begin{array}{l}\text { - } 160 \text { parent/adolescent dyads. } \\
\text { - } M \text {-age = I4.6 years, SD=1.7 } \\
\text { - } 77(48.1 \%) \text { male, } 83(51.9 \%) \text { female } \\
\text { - } 41.9 \% \text { non-Hispanic white }\end{array}$ & $\begin{array}{l}\text { - TV/video/DVD, computer or video } \\
\text { games, internet/email } \\
\text { - Parent and adolescent reported time } \\
\text { spent by adolescent on each activity. }\end{array}$ & & $x$ \\
\hline 6 & $\begin{array}{l}\text { Springer et al. } \\
(2010)^{32}\end{array}$ & USA & $\begin{array}{l}\text { - } 734 \text { children } \\
\text { - M-age = } 9.5 \text { years (at baseline) }\end{array}$ & $\begin{array}{l}\text { - TV } \\
\text { - Child reported } \\
\text { - Questions related to previous day and } \\
\text { previous weekend. }\end{array}$ & & $x$ \\
\hline 7 & $\begin{array}{l}\text { Van den Bulck } \\
\text { and Van den } \\
\text { Bergh. } \\
(2000)^{33}\end{array}$ & Belgium & $\begin{array}{l}\text { - } 519 \text { children } \\
\text { - } 261 \text { ( } 50.2 \%) \text { male, } 258 \text { (49.7\%) female } \\
\text { - Majority aged } 10 \text { to II. M-age = } 10 \text { years } \\
6 \text { months }\end{array}$ & $\begin{array}{l}\text { - TV, computer games } \\
\text { - Child reported TV/computer games } \\
\text { usage. }\end{array}$ & & $x$ \\
\hline 8 & $\begin{array}{l}\text { Warren et al. } \\
(2002)^{34}\end{array}$ & USA & $\begin{array}{l}\text { - } 321 \text { parents } \\
\text { - M-age= } 9 \text { years, age range }=1-17 \text { years } \\
\text { - } 91 \% \text { Caucasian }\end{array}$ & $\begin{array}{l}\text { - TV } \\
\text { - Not explained. }\end{array}$ & & $x$ \\
\hline 9 & $\begin{array}{l}\text { Holman and } \\
\text { Braithwaite } \\
(1982)^{35}\end{array}$ & Australia & $\begin{array}{l}\text { - } 282 \text { parents } \\
\text { - M-age of child }=3.4 \text {, age range }=3-6 \text { years. }\end{array}$ & $\begin{array}{l}\text { - TV } \\
\text { - Parental report estimate of child } \\
\text { viewing. }\end{array}$ & & $x$ \\
\hline 10 & Sun. $(2009)^{36}$ & China & $\begin{array}{l}\text { - } 1056 \text { children and adolescents } \\
\text { - } 564(53.4 \%) \text { male, } 492(46.6 \%) \text { female } \\
\text { - Age range }=6 \text { tol4 }\end{array}$ & $\begin{array}{l}\text { - TV } \\
\text { - Parent and child/adolescent reported } \\
\text { - Four questions measured child viewing. }\end{array}$ & & $x$ \\
\hline II & $\begin{array}{l}\text { Barkin et al. } \\
(2006)^{37}\end{array}$ & $\begin{array}{l}\text { USA, Canada, } \\
\text { Puerto Rico }\end{array}$ & $\begin{array}{l}\text { - } 183 \text { I parents } \\
\text { - } 54.3 \% \text { children aged } 2-5 \text { and } 45.7 \% \text { aged } \\
6-11 \text { years old. } \\
\text { - } 70 \% \text { white }\end{array}$ & $\begin{array}{l}\text { - TV and computer/video games } \\
\text { - Parent reported hours/day child TV, } \\
\text { videos, computer games, Gameboy } \\
\text { usage. }\end{array}$ & & $\mathbf{x}$ \\
\hline 12 & $\begin{array}{l}\text { Barradas et al. } \\
(2007)^{38}\end{array}$ & USA & $\begin{array}{l}\text { - } 1045 \text { parent-child dyads. } \\
520(49.8 \%) \text { boys, } 525(50.2 \%) \text { girls } \\
\text { - Age range } 10-12 \text { years }(n=334,52 \% \\
\text { female), I3-15 ( } n=355,52 \% \text { female }) \text {, } \\
\text { I6- } 18(n=346,49 \% \text { female })\end{array}$ & $\begin{array}{l}\text { - TV } \\
\text { - Parent and child reported } \\
\text { - Total TV hours = weekday TV hours } \\
\text { (X 5) + weekend TV hours. }\end{array}$ & & $x$ \\
\hline 13 & $\begin{array}{l}\text { Bauer et al. } \\
(201 \mathrm{I})^{39}\end{array}$ & USA & $\begin{array}{l}\text { - } 253 \text { parent-adolescent dyads } \\
\text { - M-age }=15.7 \text { years, age range }=14-20.3 \\
\text { - } 29 \% \text { white, } 26 \% \text { African, } 24 \% \text { Asian }\end{array}$ & $\begin{array}{l}\text { - TV } \\
\text { - Child reported 3-day PA-recall } \\
\text { completed in 30-minute blocks } \\
\text { (6am-midnight). }\end{array}$ & & $x$ \\
\hline
\end{tabular}




\section{Table I. Summary of Studies Included in the Review Including Participant Characteristics, SV Assessment Method, and Whether Parenting Styles (PS) or Parenting Practices (PP) Were Assessed continued}

\begin{tabular}{|c|c|c|c|c|c|c|}
\hline ID & Reference & Country & $\begin{array}{l}\text { Sample details and } \\
\text { child/adolescent demographics }\end{array}$ & Screen viewing measurement & PS & PP \\
\hline 14 & $\begin{array}{l}\text { Carlson et al. } \\
(2010)^{40}\end{array}$ & USA & $\begin{array}{l}\text { - } 7415 \text { children and adolescents and } 5685 \\
\text { parents. } \\
\text { - } 3840(51 \%) \text { male, } 3575 \text { (49\%) female } \\
\text { - Panel I }(n=2256 \text { aged II-15,). Panel } 2 \\
(n=5177 \text {, aged } 9-13) \text {. } \\
\text { - } 60.7 \% \text { white }\end{array}$ & $\begin{array}{l}\text { - TV and computer/video games. } \\
\text { - Child reported minutes TV, video } \\
\text { game, or computer game usage } \\
\text { on previous day. }\end{array}$ & & $\mathbf{x}$ \\
\hline 15 & $\begin{array}{l}\text { Davison et al. } \\
(2005)^{41}\end{array}$ & USA & $\begin{array}{l}\text { - I73 families (girls, mothers, and fathers). } \\
\text { - Age at } 9(M \text {-age }=9.34, S D 0.3) \text {, and II } \\
\text { (M-age }=11.34, S D=0.3) \\
\text { - All non-Hispanic white }\end{array}$ & $\begin{array}{l}\text { - TV/video } \\
\text { - Parent reported: hours/day child } \\
\text { viewed TV/videos. }\end{array}$ & & $\mathbf{x}$ \\
\hline 16 & $\begin{array}{l}\text { Hume et al. } \\
(2010)^{42}\end{array}$ & Netherlands & $\begin{array}{l}\text { - } 338 \text { adolescents } \\
\text { - M-age }=14.0, \text { SD I.I, Age range } 12-13 \\
\text { and I4-15 } \\
\text { - } 58.3 \% \text { Dutch, } 33.4 \% \text { non-Western. }\end{array}$ & $\begin{array}{l}\text { - TV } \\
\text { - Child reported TV viewing previous } \\
\text { week. }\end{array}$ & & $\mathbf{x}$ \\
\hline 17 & $\begin{array}{l}\text { Jago et al. } \\
(201 \mathrm{I})^{43}\end{array}$ & UK & $\begin{array}{l}\text { - } 43 \text { I parent-child dyads. } \\
\text { - Age range I0-II years }\end{array}$ & $\begin{array}{l}\text { - TV } \\
\text { - Parent and child reported. Single } \\
\text { question on hours/day TV viewing. }\end{array}$ & $\mathbf{x}$ & $\mathbf{x}$ \\
\hline 18 & $\begin{array}{l}\text { Lee et al. } \\
(2009)^{44}\end{array}$ & USA & $\begin{array}{l}\text { - } 1354 \text { children and adolescents } \\
\text { - } 68 \% \text { white }\end{array}$ & $\begin{array}{l}\text { - TV, computer, video games } \\
\text { - Child reported } \\
\text { - 24-hour time diaries. }\end{array}$ & & $\mathbf{x}$ \\
\hline 19 & $\begin{array}{l}\text { Padilla-Walker } \\
\text { and Coyne. } \\
(201 \mathrm{I})^{45}\end{array}$ & USA & $\begin{array}{l}\text { - } 478 \text { families (I54 single-parent and } 324 \\
\text { two-parent) } \\
\text { - } M \text {-age }=12.34, \mathrm{SD}=1.46 \\
\text { - } 230(48 \%) \text { male, } 248(52 \%) \text { female }\end{array}$ & $\begin{array}{l}\text { - TV, video games } \\
\text { - Child report of adolescents' media use. }\end{array}$ & $\mathbf{x}$ & \\
\hline 20 & $\begin{array}{l}\text { Te Velde et al. } \\
(20 \mathrm{II})^{46}\end{array}$ & Netherlands & $\begin{array}{l}\text { - } 1265 \text { children/adolescents. } \\
\text { - } 632 \text { boys, } 529 \text { girls at baseline. } \\
\text { - } 218 \text { boys, } 170 \text { girls at follow-up. } \\
\text { - M-age at follow-up }=14.7, S D=0.7\end{array}$ & $\begin{array}{l}\text { - TV } \\
\text { - Child/adolescents reported } \\
\text { - Two questions assessed TV viewing } \\
\text { (minutes/day). }\end{array}$ & & $\mathbf{x}$ \\
\hline 21 & $\begin{array}{l}\text { Vandewater } \\
\text { et al. }(2005)^{47}\end{array}$ & USA & $\begin{array}{l}\text { - } 838 \text { Parents of children aged } 6 \text { months } \\
\text { and } 6 \text { years } \\
\text { - } M \text {-age }=3.20, S D=1.82 \\
\text { - } 419(50 \%) \text { male, } 419(50 \%) \text { female }\end{array}$ & $\begin{array}{l}\text { - TV, video/DVD } \\
\text { - Parent reported viewing on previous / } \\
\text { last typical day. }\end{array}$ & & $\mathbf{x}$ \\
\hline 22 & $\begin{array}{l}\text { Griffore and } \\
\text { Phenice. } \\
(1996)^{48}\end{array}$ & USA & - I558 Children & $\begin{array}{l}\text { - TV } \\
\text { - Child reported hours on TV on typical } \\
\text { weekday and weekend day }\end{array}$ & & $\mathbf{x}$ \\
\hline 23 & $\begin{array}{l}\text { Hardy et al. } \\
(2006)^{49}\end{array}$ & Australia & $\begin{array}{l}\text { - } 974,343 \text { adolescents, } 338 \text { mothers, } \\
293 \text { fathers } \\
\text { - } M \text {-age }=12.9, S D=0.21 \\
\text { - } 483(49.6 \%) \text { male, } 491 \text { ( } 50.4 \%) \text { female }\end{array}$ & $\begin{array}{l}\text { - TV, video, DVD } \\
\text { - Child reported TV/video/DVD viewing } \\
\text { on usual week/end day. }\end{array}$ & & $\mathbf{x}$ \\
\hline 24 & $\begin{array}{l}\text { Salmon et al. } \\
(2005)^{50}\end{array}$ & Australia & $\begin{array}{l}\text { - } 927 \text { families (parents and children) } \\
\text { - M-age }=11.5, S D=0.6\end{array}$ & $\begin{array}{l}\text { - TV, electronic games, computer. } \\
\text { - Parent reported hours/minutes child } \\
\text { viewing on typical weekend. }\end{array}$ & & $\mathbf{x}$ \\
\hline 25 & $\begin{array}{l}\text { Wiecha et al. } \\
(200 \mathrm{I})^{51}\end{array}$ & USA & $\begin{array}{l}\text { - } 1197 \text { children/adolescents } \\
\text { - } 50 \% \text { male } \\
\text { - } 60 \% \text { white } \\
\text { - } 58 \% \text { grade } 7,42 \% \text { grade } 6\end{array}$ & $\begin{array}{l}\text { - TV, video, computer game. } \\
\text { - Child/adolescent reported. } \\
\text { - Assessed typical usage (hours/day) of } \\
\text { TV, video, and computer game usage. }\end{array}$ & & $\mathbf{x}$ \\
\hline 26 & $\begin{array}{l}\text { Truglio et al. } \\
(1996)^{52}\end{array}$ & USA & $\begin{array}{l}\text { - } 27 \text { I children and 'their families'. } \\
\text { - Children: within } 3 \text { months of } 3^{\text {rd }}(n=160) \\
\text { and } 5^{\text {th }} \text { birthday }(n=166) \text {, at baseline. }\end{array}$ & $\begin{array}{l}\text { - TV (entertainment viewing) } \\
\text { - Parent diaries (I week in spring, } \\
\text { I week in fall for } 2 \text { study years; } \\
5 \text { diaries in total). }\end{array}$ & & $\mathbf{x}$ \\
\hline
\end{tabular}




\begin{tabular}{|c|c|c|c|c|c|c|}
\hline ID & Reference & Country & $\begin{array}{l}\text { Sample details and } \\
\text { child/adolescent demographics }\end{array}$ & Screen viewing measurement & PS & PP \\
\hline 27 & $\begin{array}{l}\text { van Zutphen } \\
\text { et al. }(2007)^{53}\end{array}$ & Australia & $\begin{array}{l}\text { - } 1926 \text { children } \\
\text { - } \mathrm{M} \text {-age }=8.0 \text { years, } \mathrm{SD}=0.05 \text {, Age range }= \\
3.5-12.5 \text { years } \\
\text { - } 940(48.8 \%) \text { male, } 986(51.2 \%) \text { female }\end{array}$ & $\begin{array}{l}\text { - TV, video. } \\
\text { - Parent reported child video/TV viewing } \\
\text { on previous day (minutes) and usual } \\
\text { viewing. }\end{array}$ & & $x$ \\
\hline 28 & $\begin{array}{l}\text { Gable and } \\
\text { Lutz. }(2000)^{54}\end{array}$ & USA & $\begin{array}{l}\text { - } 65 \text { parent and child pairs } \\
\text { - } 28(43 \%) \text { male, } 37(57 \%) \text { female } \\
\text { - } 75 \% \text { white } \\
\text { - } \text { M-age }=8.1 \text { years, age range }=6-10.9\end{array}$ & $\begin{array}{l}\text { - TV } \\
\text { Parent reported average number of } \\
\text { hours/day child spent watching TV } \\
\text { (don't watch TV, }<\text { I hour/day, } \\
\text { I-2 hours/day, } 2-3 \text { hours/day, } \\
>3 \text { hours/day). }\end{array}$ & $x$ & \\
\hline 29 & $\begin{array}{l}\text { Shin and Huh. } \\
(2011)^{55}\end{array}$ & USA & $\begin{array}{l}\text { - II02 parent and adolescent pairs } \\
\text { - M-age }=15 \text {, age range }=12-17 \\
\text { - } 51 \% \text { males, } 49 \% \text { females }\end{array}$ & $\begin{array}{l}\text { - Video gaming } \\
\text { - Adolescent and parent reported } \\
\text { frequency of playing on a 7-point scale. }\end{array}$ & & $\mathbf{x}$ \\
\hline
\end{tabular}

M-age, mean age; SD, standard deviation; PA, physical activity.

\section{Parenting Practices}

A summary of the parenting practices that were assessed in each of the studies is presented in Table $3,27-44,46-53,55$ with an overview of the practices presented in Table 4. ${ }^{27-44,46-53,55}$ As noted above, seven rules were reported, with the following frequencies across studies: (1) Limits on total time ( $n$ studies $=23$ ); (2) limits on time of day $(n=7)$; (3) content restriction $(n=11)$; (4) mealtime rules $(n=2)$; (5) parental supervision $(n=3)$; (6) contingent SV $(n=3)$; and (7) no-TV policy $(n=1)$. The limit-based rules were subdivided into studies that focused on TV, $\mathrm{DVD} / \mathrm{VCR}, \mathrm{PC}$, and video games. Limits on total TV time and on the time of day children watch TV were most frequently examined. Two nonrule-based practices were reported-co-viewing $(n=6)$ and encouragement to view $(n=2)$. Eleven of the studies had child reports of parenting practices, 11 had parent reports, and 5 used both parent and child reports. In many studies, analyses were conducted on a composite exposure variable that consisted of multiple different types of parenting practice and, as such, may not use the same terms that are presented above. Two studies ${ }^{32,43}$ made reference to a published scale. ${ }^{59-62}$

\section{Quality of Measures Used}

Outcome measures. A summary of whether any information was provided on the reliability, internal consistency of the SV outcome measure is shown in Table 5. Of the 29 studies that were included in the review, only 12 $(41.4 \%)^{27,28,31,39,42,43,45,46,49-52}$ provided any justification for the selection of the SV outcome. Of the studies that did provide information, five reported information on testretest reliability based on their own data $28,31,46,49,50$ and four reported information about criterion validity when compared to another measure reported in the text. ${ }^{28,43,51,52}$ In total, nine studies $27,28,31,39,42,43,45,46,52$ provided a reference to support their scales with 12 different references provided. ${ }^{63-74}$

\section{Table 2. Summary of Parenting Style Measures}

\begin{tabular}{|c|c|c|c|}
\hline ID & Study reference & Respondent & $\begin{array}{l}\text { Items assessed under label 'parenting style' } \\
\text { and associated reference (if provided) }\end{array}$ \\
\hline 17 & Jago et al. $(20 \mathrm{II})^{43}$ & Child & $\begin{array}{l}\text { Maternal parenting style, using } 30 \text {-item version of Children's Report of Parent Behavior } \\
\text { Inventory. } \\
\text { Mean scores classify parents as: (I) Authoritative (high acceptance/high control), (2) } \\
\text { Authoritarian (low acceptance/high control), and (3) Permissive (high acceptance/low control). }\end{array}$ \\
\hline 19 & $\begin{array}{l}\text { Padilla-Walker and Coyne. } \\
(201 \mathrm{I})^{45}\end{array}$ & Parent & $\begin{array}{l}\text { Frequency parent adopts behaviors relating to authoritative parenting: classified as } \\
\text { connection, regulation, and autonomy. }\end{array}$ \\
\hline 28 & Gable and Lutz. $(2000)^{54}$ & Parent & $\begin{array}{l}\text { Two subscales (authoritative and authoritarian) of the CRPR. Authoritative parenting } \\
\text { reflects positive child guidance, enjoyment in the child, encouragement of child } \\
\text { autonomy, and affectionate behavior. Authoritarian parenting styles are controlling, } \\
\text { prohibitive, and anxiety-inducing. }\end{array}$ \\
\hline
\end{tabular}

CRPR, Child-Rearing Practices Report. 


\section{Table 3. Summary of Parenting Practices That Were Assessed}

\begin{tabular}{|c|c|c|c|c|}
\hline ID & Study & Respondent & Parenting practices assessed & $\begin{array}{c}\text { Summary of measure and associated } \\
\text { external reference (if reported) }\end{array}$ \\
\hline I & $\begin{array}{l}\text { Barr et al. } \\
(2010)^{27}\end{array}$ & Parent & $\begin{array}{l}\text { - Limit on total viewing time } \\
\text { - Content restriction } \\
\text { - Co-viewing } \\
\text { - No-TV policy }\end{array}$ & $\begin{array}{l}\text { - Restrictions on time of TV use. } \\
\text { - Co-viewing: How often parent was in room } \\
\text { when infant viewed TV }\end{array}$ \\
\hline 2 & $\begin{array}{l}\text { He et al. } \\
(2010)^{28}\end{array}$ & $\begin{array}{l}\text { Parent and } \\
\text { child }\end{array}$ & - Rules (limit on total viewing / use) & $\begin{array}{l}\text { - Perceived rules (week/end) for: limit TV, limit } \\
\text { videogame, limit computer use for homework } \\
\text { (and non-homework). }\end{array}$ \\
\hline 3 & $\begin{array}{l}\text { Lin and Atkin. } \\
(1989)^{29}\end{array}$ & Child & $\begin{array}{l}\text { - Program/video content restrictions } \\
\text { - Encouragement to watch certain content } \\
\text { - Limit on total viewing/video hire } \\
\text { - Limit on time of day child can view TV } \\
\text { - Limit on frequency child views with } \\
\text { friends }\end{array}$ & $\begin{array}{l}\text { - 'Viewing rules' assessed by three items: (a) time } \\
\text { child is allowed to view TV, (b) content, (c) how } \\
\text { late child views TV. } \\
\text { - VCR rules assessed by: (a) frequency child rents } \\
\text { video, (b) content, (c) frequency views videos at } \\
\text { friend's house, (d) how often child could rent } \\
\text { video with friend. }\end{array}$ \\
\hline 4 & $\begin{array}{l}\text { Patriarca et al. } \\
(2009)^{30}\end{array}$ & Child & $\begin{array}{l}\text { - Control / supervision of TV viewing, } \\
\text { videogame, and computer use. }\end{array}$ & $\begin{array}{l}\text { - Do parents control or supervise TV viewing, } \\
\text { playing videogames, using computer (yes/no). }\end{array}$ \\
\hline 5 & $\begin{array}{l}\text { Ramirez et al. } \\
(2011)^{31}\end{array}$ & $\begin{array}{l}\text { Parent and } \\
\text { child }\end{array}$ & $\begin{array}{l}\text { - TV and computer rules } \\
\text { - Video games - total rules } \\
\text { - Limit on total viewing } \\
\text { - Limit on time child can view TV } \\
\text { - Contingent SV }\end{array}$ & $\begin{array}{l}\text { - Agree/disagree with } 7 \text { items on rules: Not too } \\
\text { much TV/DVDs, No TV/DVD before homework, } \\
\text { No TV/DVD while doing homework, <2 hour } \\
\text { TV/DVD/day, No computer before HW, only Ihr } \\
\text { computer/d, no internet without permission, } \\
\text { no TV/computer unless exercised first. }\end{array}$ \\
\hline 6 & $\begin{array}{l}\text { Springer et al. } \\
(2010)^{32}\end{array}$ & Child & - Limit on total TV & $\begin{array}{l}\text { - Primary independent variable assessed parental } \\
\text { rules for child TV viewing } 59,60,62\end{array}$ \\
\hline 7 & $\begin{array}{l}\text { Van den Bulck } \\
\text { et al. }(2000)^{33}\end{array}$ & Child & $\begin{array}{l}\text { - Content restrictions } \\
\text { - Limit on total viewing } \\
\text { - Limits on time of day } \\
\text { - Co-viewing/ playing computer games }\end{array}$ & $\begin{array}{l}\text { - Parental guidance behavior with regard to TV } \\
\text { viewing and computer usage. For } 16 \text { questions, } \\
\text { child responded 'never' to 'often'. 'Restrictive } \\
\text { guidance' includes TV and computer game } \\
\text { content restrictions and limits on TV and } \\
\text { computer game time. }\end{array}$ \\
\hline 8 & $\begin{array}{l}\text { Warren et al. } \\
(2002)^{34}\end{array}$ & Parent & $\begin{array}{l}\text { - Co-viewing } \\
\text { - Content restrictions } \\
\text { - Limits on total viewing } \\
\text { - Limits on time of day }\end{array}$ & $\begin{array}{l}\text { - Likert-scale items measured frequency of } \\
\text { co-viewing, restrictions, and discussion of TV } \\
\text { viewing with child. }\end{array}$ \\
\hline 9 & $\begin{array}{l}\text { Holman et al. } \\
(1982)^{35}\end{array}$ & Parent & - Content restriction & $\begin{array}{l}\text { - Parenting control of TV measured through } \\
\text { two items assessing programme suitability } \\
\text { and selection. }\end{array}$ \\
\hline 10 & Sun. $(2009)^{36}$ & Child & $\begin{array}{l}\text { - Content restriction } \\
\text { - Co-viewing } \\
\text { - Limit on total time }\end{array}$ & $\begin{array}{l}\text { - Control over child TV viewing time, and content } \\
\text { control was averaged to create 'restrictive } \\
\text { mediation' variable. Co-viewing is measured by } \\
\text { one question asking child how often they view } \\
\text { with parents each week. }\end{array}$ \\
\hline 11 & $\begin{array}{l}\text { Barkin et al. } \\
(2006)^{37}\end{array}$ & Parent & $\begin{array}{l}\text { - Content restrictions } \\
\text { - Limits on times of viewing } \\
\text { - Limits on time of day }\end{array}$ & $\begin{array}{l}\text { - How often in the past month have you restricted } \\
\text { use, explained content, or allowed unlimited use. }\end{array}$ \\
\hline 12 & $\begin{array}{l}\text { Barradas et al. } \\
(2007)^{38}\end{array}$ & $\begin{array}{l}\text { Parent and } \\
\text { child }\end{array}$ & - Limits on total time & $\begin{array}{l}\text { - Frequency parent set rules on amount of time } \\
\text { child views TV and parent encourages daughter } \\
\text { to view less TV }\end{array}$ \\
\hline 13 & $\begin{array}{l}\text { Bauer et al. } \\
(201 \mathrm{I})^{39}\end{array}$ & Parent & - Limits on total viewing & - TV limits assessed with single question \\
\hline 14 & $\begin{array}{l}\text { Carlson et al. } \\
(2010)^{40}\end{array}$ & $\begin{array}{l}\text { Parent and } \\
\text { child }\end{array}$ & $\begin{array}{l}\text { - Limits on total time } \\
\text { - Limits and rules on total videogame use }\end{array}$ & $\begin{array}{l}\text { - Child-report: Parents have rules for TV viewing } \\
\text { time and videogames. } \\
\text { - Parent-report: How often they set limits on time } \\
\text { child views TV/videogames). }\end{array}$ \\
\hline
\end{tabular}




\section{Table 3. Summary of Parenting Practices That Were Assessed continued}

\begin{tabular}{|c|c|c|c|c|}
\hline ID & Study & Respondent & Parenting practices assessed & $\begin{array}{l}\text { Summary of measure and associated } \\
\text { external reference (if reported) }\end{array}$ \\
\hline 15 & $\begin{array}{l}\text { Davison et al. } \\
(2005)^{41}\end{array}$ & Parent & $\begin{array}{l}\text { - Co-viewing (TV and videos) } \\
\text { - Limits on total time (restriction of } \\
\text { access) }\end{array}$ & $\begin{array}{l}\text { - Co-viewing = frequency parents watched } \\
\text { TV/videos with daughter or as family } \\
\text { - Parents separately report limits on daughter's } \\
\text { TV viewing (age II): do not limit -to- only select } \\
\text { programs or no TV at all. }\end{array}$ \\
\hline 16 & $\begin{array}{l}\text { Hume et al. } \\
(2010)^{42}\end{array}$ & Child & $\begin{array}{l}\text { - Rules (limits) on total time } \\
\text { - Rules (limits) on time of day }\end{array}$ & $\begin{array}{l}\text { Dichotomous (yes/no) scale; parent rules on } \\
\text { total TV viewing, and rules about when child can } \\
\text { view. Items summed to create scale of TV } \\
\text { viewing rules }\end{array}$ \\
\hline 17 & $\begin{array}{l}\text { Jago et al. } \\
(2011)^{43}\end{array}$ & Child & $\begin{array}{l}\text { - Limits on total viewing, video/computer } \\
\text { game use and non-homework use }\end{array}$ & $\begin{array}{l}\text { Limit on TV, comp/videogame, and computer } \\
\text { time for non-homework. 4-point scale. Combined } \\
\text { to form three categories: (I) both parents high; } \\
\text { (2) I high, I low; (3) both parents low. }{ }^{61}\end{array}$ \\
\hline 18 & $\begin{array}{l}\text { Lee et al. } \\
(2009)^{44}\end{array}$ & Child & - Limits on total time & $\begin{array}{l}\text { - Items summed to form one variable. How } \\
\text { much time child watches TV, limits on staying } \\
\text { up at night. }\end{array}$ \\
\hline 20 & $\begin{array}{l}\text { Te Velde et al. } \\
(201 \mathrm{I})^{46}\end{array}$ & Child & $\begin{array}{l}\text { - Limits on total time } \\
\text { - Limits on time of day }\end{array}$ & $\begin{array}{l}\text { - Rules about time of TV viewing and total } \\
\text { viewing time (both Yes/No). }\end{array}$ \\
\hline 21 & $\begin{array}{l}\text { Vandewater } \\
\text { et al. }(2005)^{47}\end{array}$ & Parent & $\begin{array}{l}\text { - Content restriction } \\
\text { - Limit on total time }\end{array}$ & $\begin{array}{l}\text { - Rules about viewing TV and rules about content } \\
\text { (both Yes/No). }\end{array}$ \\
\hline 22 & $\begin{array}{l}\text { Griffore et al. } \\
(1996)^{48}\end{array}$ & Child & - Rules on total time. & - Rules about viewing TV. \\
\hline 23 & $\begin{array}{l}\text { Hardy et al. } \\
(2006)^{49}\end{array}$ & $\begin{array}{l}\text { Parent } \\
\text { and child }\end{array}$ & $\begin{array}{l}\text { - Meal time } \\
\text { - Contingent SV } \\
\text { - Parental supervision }\end{array}$ & $\begin{array}{l}\text { - Parent ensures child does not view too much } \\
\text { TV (disagree, neutral, agree). } \\
\text { - Restrictions on child TV viewing; prohibit TV } \\
\text { until homework is done; and restrict TV } \\
\text { at meals. }{ }^{76} \\
\text { - Can child view as much TV as they like, do they } \\
\text { have limits on TV, always have limits, or do not } \\
\text { view TV }\end{array}$ \\
\hline 24 & $\begin{array}{l}\text { Salmon et al. } \\
(2005)^{50}\end{array}$ & Parent & $\begin{array}{l}\text { - Limits on total time on TV, electronic } \\
\text { games, and computer } \\
\text { - Contingent SV } \\
\text { - Content restriction } \\
\text { - Meal time }\end{array}$ & $\begin{array}{l}\text { - For each screen-based behavior; restrictions } \\
\text { over time child spends in each activity. 4-scale } \\
\text { response 'don't know/doesn't apply' to } \\
\text { 'very often'. }\end{array}$ \\
\hline 25 & $\begin{array}{l}\text { Wiecha et al. } \\
(200 I)^{51}\end{array}$ & Child & - Limit on total time & $\begin{array}{l}\text { - Limit setting defined by response to four } \\
\text { questions. Responses dichotomized as 'no limits' } \\
\text { or 'parents place limits'. }\end{array}$ \\
\hline 26 & $\begin{array}{l}\text { Truglio et al. } \\
(1996)^{52}\end{array}$ & Parent & $\begin{array}{l}\text { - Content restriction } \\
\text { - Limit on total time } \\
\text { - Encouragement to view } \\
\text { - Parental supervision }\end{array}$ & $\begin{array}{l}\text { - Encouragement to view measured on } 5 \text {-point } \\
\text { Likert scale ( } I=\text { never, } 5=\text { very often). Parent } \\
\text { regulation measured by } 3 \text { items. } \\
\text { - Regulation of TV use measured by independent } \\
\text { child viewing, time limits, programs restrictions. }\end{array}$ \\
\hline 27 & $\begin{array}{l}\text { van Zutphen } \\
\text { et al. }(2007)^{53}\end{array}$ & Parent & - Limits on total TV and DVD time & $\begin{array}{l}\text { - Rules limiting hours child views TV/videos and } \\
\text { tightness of rules } \\
\text { - Rules regarding total time child can view TV, } \\
\text { and tightness of rule enforcement. }\end{array}$ \\
\hline 29 & $\begin{array}{l}\text { Shin and Huh. } \\
(20 \mathrm{II})^{55}\end{array}$ & Parent & $\begin{array}{l}\text { - Limit on total viewing time } \\
\text { - Content restriction } \\
\text { - Co-viewing (video games) }\end{array}$ & $\begin{array}{l}\text { - Co-playing, checking ratings and stopping child } \\
\text { from playing video games was measured on } \\
\text { 4-point Likert scale. }\end{array}$ \\
\hline
\end{tabular}




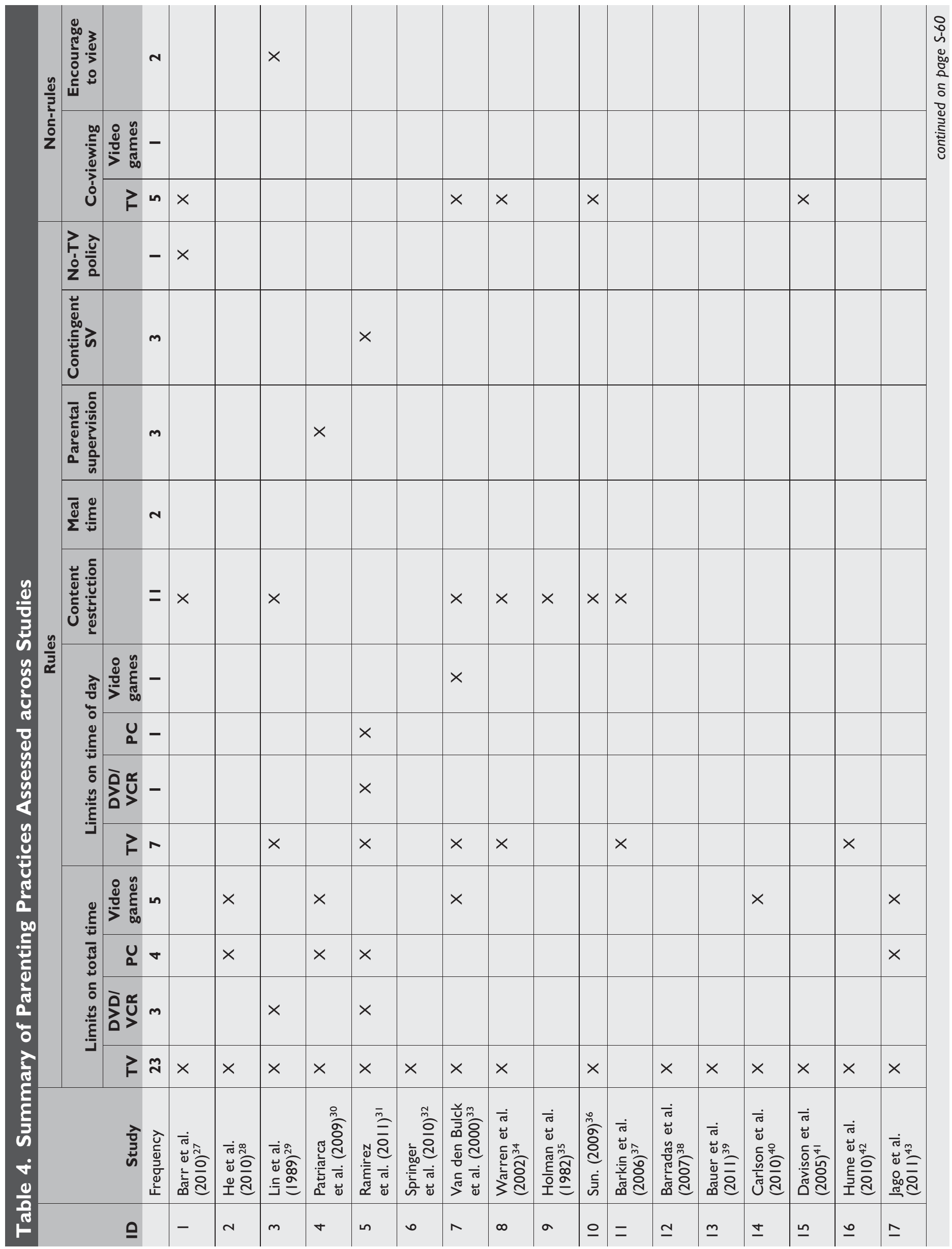




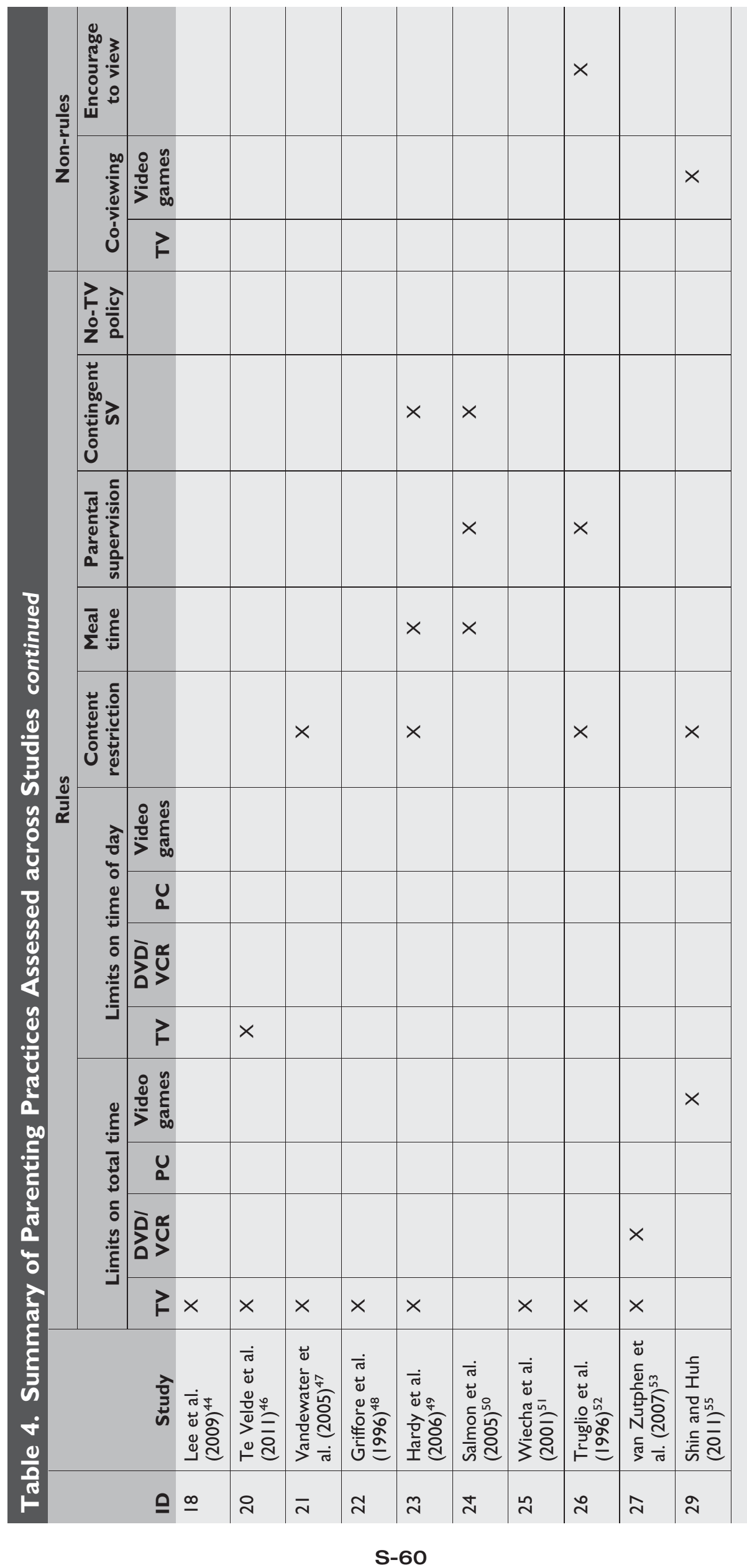




\section{Table 5. Reported Reliability and Validity of Screen-Viewing Measures and Link} to External Validation/Reliability Information

\begin{tabular}{|c|c|c|c|}
\hline ID & Study & Information reported on the reliability and or validity of SV measure & Reference \\
\hline I & Barr et al. $(2010)^{27}$ & - & 69,72 \\
\hline 2 & He et al. $(2010)^{28}$ & $\begin{array}{l}\text { ICC range }=0.5 \text { to } 0.8 \text { when compared with Activity Diary Method. 2-week test retest } \\
I C C=0.98 \text {. }\end{array}$ & 63 \\
\hline 3 & Lin and Atkin. $(1989)^{29}$ & - & - \\
\hline 4 & Patriarca et al. $(2009)^{30}$ & - & - \\
\hline 5 & Ramirez et al. $(201 \mathrm{I})^{3 \mathrm{I}}$ & $\begin{array}{l}\text { 2-week test retest. Weekday }(\mathrm{ICC}=0.44,95 \% \mathrm{Cl}=0.56,0.57) \text {, weekend }(\mathrm{ICC}=0.46 \text {, } \\
95 \% \mathrm{Cl}=0.52,0.63)\end{array}$ & 64-66 \\
\hline 6 & Springer et al. $(2010)^{32}$ & - & - \\
\hline 7 & $\begin{array}{l}\text { Van den Bulck et al. } \\
(2000)^{33}\end{array}$ & - & - \\
\hline 8 & Warren et al. $(2002)^{34}$ & 一 & - \\
\hline 9 & Holman et al. $(1982)^{35}$ & - & - \\
\hline 10 & Sun. $(2009)^{36}$ & - & - \\
\hline 11 & Barkin et al. $(2006)^{37}$ & - & - \\
\hline 12 & Barradas et al. $(2007)^{38}$ & - & - \\
\hline 13 & Bauer et al. $(201 \mathrm{I})^{39}$ & - & 73,74 \\
\hline 14 & Carlson et al. $(2010)^{40}$ & - & - \\
\hline 15 & Davison et al. $(2005)^{4 l}$ & - & - \\
\hline 16 & Hume et al. $(2010)^{42}$ & Three TV viewing habit strength variables created (Cronbach $\alpha=0.82$ ). & 67,68 \\
\hline 17 & Jago et al. $(201 \mathrm{I})^{43}$ & $\begin{array}{l}\text { Assessment of TV viewing with single question shown to correlate }(r=0.60) \text { with } \\
10 \text { days of TV diaries. }\end{array}$ & 69 \\
\hline 18 & Lee et al. (2009) ${ }^{44}$ & - & - \\
\hline 19 & $\begin{array}{l}\text { Padilla-Walker et al. } \\
(20 I \mathrm{I})^{45}\end{array}$ & Average use of videogames and TV $(\alpha=0.65)$ & 70 \\
\hline 20 & Te Velde et al. $(20 \mathrm{II})^{46}$ & $\begin{array}{l}\text { Previous reliability study ( } n=49 \text { children test-retest, } 2 \text {-week interval) showed good } \\
\text { reliability }(I C C=0.78)\end{array}$ & 71 \\
\hline 21 & $\begin{array}{l}\text { Vandewater et al. } \\
(2005)^{47}\end{array}$ & - & - \\
\hline 22 & Griffore et al. $(1996)^{48}$ & - & - \\
\hline 23 & Hardy et al. $(2006)^{49}$ & $\begin{array}{l}\text { Two-week test-retest }(n=140) \text { with good-excellent repeatability (percent } \\
\text { agreement }=70-99 \%) .\end{array}$ & - \\
\hline 24 & Salmon et al. $(2005)^{50}$ & $\begin{array}{l}\text { Test-retest reliability for proxy-reported time screen viewing behaviors (ICC) ranged } \\
\text { from } 0.6 \text { to } 0.8 \text {. }\end{array}$ & - \\
\hline 25 & Wiecha et al. $(200 \mathrm{I})^{51}$ & $\begin{array}{l}\text { Validation ( } n=53 \text { students) correlation of } r=0.54 \text { between survey and the recall for } \\
\text { TV with equivalent means. }\end{array}$ & - \\
\hline 26 & Truglio et al. $(1996)^{52}$ & Comparison of diaries and videotapes was 0.84 for preschoolers' viewing. & 69 \\
\hline 27 & $\begin{array}{l}\text { van Zutphen et al. } \\
(2007)^{53}\end{array}$ & - & - \\
\hline 28 & Gable and Lutz $(2000)^{54}$ & - & - \\
\hline 29 & Shin and Huh $(201 \mathrm{I})^{55}$ & - & - \\
\hline
\end{tabular}




\section{Parenting Styles and Practices}

A summary of whether information was provided on the reliability, factor structure, internal consistency, or reference to an external study is presented in Table 6. Of the three studies that assessed parenting styles, ${ }^{43,45,54}$ two reported information on the internal consistency of the measures ${ }^{45,54}$ and one provided information on differences in assessments of the scale that were conducted 2 weeks apart. ${ }^{43}$ Of the 12 studies that provided any support for selection of the parenting practice measure, five studies directly reported internal consistency information, ${ }^{29,34,36,42,44}$ five studies referred the reader to data presented in a previous article, ${ }^{32,34,43,49,52}$ four studies provided reliability information, ${ }^{31,43,49,50}$ and two studies provided information on the factor structure of the items. ${ }^{33,34}$ In total, nine external references were provided to support the inclusion of scales. ${ }^{56,57,59-61,75-78}$ Combining information from Tables 5 and 6 indicates that only six $(20.7 \%)$ of the studies included in the review provided any information on the reliability, validity, factor structure, or internal consistency of both the outcome and exposure measures. ${ }^{31,42,43,49,50,52}$

\section{Associations between Parenting and SV}

Parenting styles. Three studies examined the associations between parenting styles and SV. ${ }^{43,45,54}$ The studies examined different parenting constructs and employed different methodologies making it impossible to ascertain whether there are patterns of association between parenting styles and youth SV.

Parenting practices. The results of the 27 studies that examined parenting practice $27-44,46-53,55$ are presented, stratified by behavior (TV, computer, or video) and parenting practice in Table 7 . There were 21 studies that examined the association between TV viewing/watching DVDs and parental rules. ${ }^{27-33,35-38,40,42,43,46-50,52,53}$ The analysis of these studies used analysis of variance, chisquared analysis, logistic regression, linear regression, and tests of trends. Similarly, the results for TV/DVD co-viewing used Spearman rank order correlations, logistic regression, and linear regression. The variety in analytical frameworks was repeated for each of the other subgroup analyses that are presented in Table 7. Because the studies included in each subgroup are also heterogeneous in terms of participants, setting, and measures, combining the data from across studies to draw any conclusions about patterns in the data is very difficult and a formal meta-analysis is not possible. Similarly, presenting findings as positive, negative, or null associations based on an arbitrary $p$ value of $<0.05$ could also be misleading because both the type of analysis and sample size affects the $p$ value obtained, which could lead to misinterpretation of potentially important findings. ${ }^{79}$

Although we acknowledge the inherent difficulty in combining data from heterogeneous studies, it would be helpful to have an impression of the range of findings and the strength of those findings that have been reported. This overview could be used to identify research gaps that could be further examined in future studies. As such, we have rated the strength of evidence for each of the analyses that were conducted across studies as either: (1) Not reported; (2) little evidence $(p>0.05)$; (3) some evidence $(p<0.05)$; (4) increasing evidence $(p<0.01)$; and (5) strong evidence $(p<0.001) .{ }^{79}$ (Please note that some articles included multiple analyses and all tests were included in this summary.) The percent of studies in each evidence category are then shown graphically in Figure 2 for each of the ten subgroups of studies that were presented in Table 7. The number of studies included in each subgroup is also presented to facilitate an interpretation of how associations may be a function of the number of analyses conducted. A visual inspection of the figure indicates that across the nine different subgroup analyses there are no clear patterns of all strong evidence or all little evidence, suggesting that the evidence for each parenting practice and youth SV is inconsistent.

\section{Discussion}

The data presented in this systematic review indicate that there were 29 studies $^{27-55}$ that examined associations between parenting styles or parenting practices and youth $\mathrm{SV}$ that met the review inclusion criteria. Only three ${ }^{43,45,54}$ of the 29 studies examined aspects of general parenting constructs in relation to SV. The review has highlighted that the majority of the studies have used SV outcome measures and/or parenting style/practice exposure measures for which there is little or no information about either validity or reliability. Moreover, there is considerable variability in terms of study design, participants, and analytical methods, which makes synthesizing the data from across studies very difficult. However, the general patterns of association and strength of evidence for each parenting practice are mixed, suggesting that while the parenting practices identified (i.e., limits on total time, limits on time of day, content restriction, mealtime rules, parental supervision, contingent SV, no-TV policy, co-viewing, and encouragement) could be important influences on youth $\mathrm{SV}$, it is not currently possible to ascertain what parenting practices interventions should be targeted as part of future SV interventions.

Media-related parenting practices were reported by the child, the parent, or by both parent and child. While it is possible to make rational arguments for either child or parent reports based on the age of the child or the topic being assessed, it is important to recognize that child and parent reports provide different information. If the child is sufficiently cognitively advanced to provide "reliable" information, his or her report will represent their perception of their parent's behaviors. Conversely, the parent report will provide an indication of the behavior that the parent believes they are adopting. The utility of each report may be dependent on how the information will be used. ${ }^{43}$ For example, a parent's report of their media-parenting strategies might be particularly useful if an intervention 
Table 6. Summary of Reliability, Internal Consistency, and Factor Structure for Parenting Style and Practice Measures

\section{Parenting styles}

\begin{tabular}{c|l|}
\hline ID & \multicolumn{1}{|c|}{ Study } \\
I7 & Jago et al. $(20 \mathrm{II})^{43}$ \\
\hline I9 & Padilla-Walker et al. $(20 \mathrm{II})^{45}$ \\
\hline 28 & Gable and Lutz $(2000)^{54}$ \\
\hline
\end{tabular}

Reliability and/or validity of measure

TRT: Paired-sample $t$-test suggest no mean difference in scores one week apart $(p>0.05)$.

References

I: Maternal connection $(\alpha=0.78)$, maternal regulation $(\alpha=0.82)$, and maternal autonomy $(\alpha=0.75)$.

61

Paternal connection $(\alpha=0.78)$, paternal regulation $(\alpha=0.84)$, and paternal autonomy $(\alpha=0.79)$.

I: Cronbach's alpha for the Child Rearing Practices Report's (CRPR) subscales (authoritative, authoritarian) were $\alpha=0.7 \mathrm{I}$ and $\alpha=0.69$, respectively.

Parenting practices

\begin{tabular}{|c|c|c|c|}
\hline ID & Study & Reliability and/or validity of measure & \\
\hline I & Barr et al. $(2010)^{27}$ & - & - \\
\hline 2 & He et al. $(2010)^{28}$ & - & - \\
\hline 3 & Lin et al. $(1989)^{29}$ & I: Internal consistency for viewing rules $(\alpha=.74)$ & - \\
\hline 4 & Patriarca et al. $(2009)^{30}$ & - & - \\
\hline 5 & Ramirez et al. $(20 \mathrm{II})^{3 !}$ & $\begin{array}{l}\text { TRT: Parental test-retest on rules }(k \text { range }=0.44-0.70) \text { Adolescent test-retest on rules }(k \\
\text { range }=0.43-0.61) \text { Agreement between parent and child on individual rules was low } \\
(k=0.28,0.47,0.45,0.44,0.49,0.17,0.53,0.00)\end{array}$ & \\
\hline 6 & Springer et al. $(2010)^{32}$ & - & 59,60 \\
\hline 7 & Van den Bulck et al. $(2000)^{33}$ & $\begin{array}{l}\text { F: Exploratory factor analysis of restrictive, evaluative and unfocused TV guidance (Cronbach } \\
\alpha \text { range }=0.55-0.86) \text { and PC-game guidance }(\alpha \text { range }=0.80-0.88) \text { for mothers and fathers. }\end{array}$ & - \\
\hline 8 & Warren et al. $(2002)^{34}$ & $\begin{array}{l}\text { F: Principal component analysis with Varimax rotation: confirmed existence of three } \\
\text { components: (a) restrictive mediation, (b) co-viewing, (c) instructive mediation. Three-factor } \\
\text { solution accounted for } 60.7 \% \text { of variance } \\
\text { I: Restrictive mediation }(\alpha=0.80) \text {, co-viewing }(\alpha=0.79) \text {, and instructive mediation }(\alpha=0.88) \\
\text { had acceptable internal consistency. }\end{array}$ & 75 \\
\hline 9 & Holman et al. $(1982)^{35}$ & - & - \\
\hline 10 & Sun. $(2009)^{36}$ & I: Restrictive mediation $(\alpha=0.6 \mathrm{I})$ and 'Instructive' mediation $(\alpha=0.90)$. & - \\
\hline II & Barkin et al. $(2006)^{37}$ & - & - \\
\hline 12 & Barradas et al. $(2007)^{38}$ & - & - \\
\hline 13 & Bauer et al. $(201 \mathrm{I})^{39}$ & - & - \\
\hline 14 & Carlson et al. $(2010)^{40}$ & - & - \\
\hline 15 & Davison et al. $(2005)^{41}$ & - & - \\
\hline 16 & Hume et al. $(2010)^{42}$ & I: Internal consistency for two item parental rules $(\alpha=0.67)$. & - \\
\hline 17 & Jago et al. $(201 \mathrm{I})^{43}$ & TRT: Paired-sample $t$-test: No difference in scores at I week apart $(p>0.05)$. & 56,57 \\
\hline 18 & Lee et al. (2009) ${ }^{44}$ & I: Internal consistency of 7 items $(\alpha=0.76)$. & - \\
\hline 20 & Te Velde et al. $(201 \mathrm{I})^{46}$ & - & - \\
\hline 21 & Vandewater et al. $(2005)^{47}$ & - & - \\
\hline 22 & Griffore et al. (1996) ${ }^{48}$ & - & - \\
\hline 23 & Hardy et al. $(2006)^{49}$ & TRT: Reliability reported in previous study & 76 \\
\hline 24 & Salmon et al. $(2005)^{50}$ & TRT: Restriction two week test-retest ICC range $=0.7 \mathrm{I}-0.88$, Rules, ICC range $=0.77-0.90$. & - \\
\hline 25 & Wiecha et al. $(200 I)^{51}$ & - & - \\
\hline 26 & Truglio et al. $(1996)^{52}$ & - & 77 \\
\hline 27 & van Zutphen et al. $(2007)^{53}$ & - & - \\
\hline 29 & Shin and Huh. $(2011)^{55}$ & - & - \\
\hline
\end{tabular}

TRT, Test-retest reliability; I, internal consistency; F, factor structure; ICC, intraclass correlation; $\alpha=$ Cronbach alpha; $k$, Cohen measure of agreement. 


\section{Table 7. Summary of Results of Associations between Parenting Practices and Screen-Viewing

\begin{tabular}{l|l|lr} 
ID & Study & Findings
\end{tabular}

\section{TV and DVD / VCR: Rules}

\begin{tabular}{|c|c|c|}
\hline I & Barr et al. $(2010)^{27}$ & $\begin{array}{l}\text { One-way ANOVA: No association between TV usage of those with no TV policy }(n=20 I, M=I \text { hour } \\
\text { I minute) and those who did not have one }(n=22, M=I \text { hour II minute). (ANOVA statistics } \\
\text { not reported.) }\end{array}$ \\
\hline 2 & He et al. $(2010)^{28}$ & $\begin{array}{l}\text { Chi-squared test: Children with total time rules more likely to have screen time }<2 \text { hours/day than } \\
\geq 2 \text { hours/day: } 73.2 \% \text { of children who had such rules had screen time }<2 \text { hours/day compared with } \\
61.3 \% \text { who reported } \geq 2 \text { hours/day }(p<0.05) \text {. }\end{array}$ \\
\hline 3 & Lin and Atkin. $(1989)^{29}$ & $\begin{array}{l}\text { Correlation: Viewing rules positively associated with child viewing }(r=0.1 \mathrm{I} ; p \leq 0.05) \\
\text { VCR rules positively associated with VCR use }(r=0.10 ; p \leq 0.05) \text { and child viewing }(r=0.09 ; p=0.09) \text {. }\end{array}$ \\
\hline 4 & Patriarca et al. $(2009)^{30}$ & $\begin{array}{l}\text { Multivariate logistic regression: Parent control over TV viewing negatively associated with child SV } \\
\geq 2 \text { hours/day }(\mathrm{OR}=0.93 ; 95 \% \mathrm{Cl}=0.68, \mathrm{I} .27 ; p=0.66) \\
\text { Multivariate linear regression: Negative association between parent control in TV viewing and whether } \\
\text { child SV } \geq 2 \text { hours/day }(\beta=0.04 ; t=0.01 ; p=0.99) \text {. }\end{array}$ \\
\hline 5 & Ramirez et al. $(20 \mathrm{II})^{3 \mathrm{I}}$ & $\begin{array}{l}\text { Multiple regression: Child report: TV viewing negatively associated with TV rules, }(B=0.18 ; \mathrm{SE}=0.07 ; \\
\beta=-0.22 ; p<0.0 \mathrm{I}) \text {. } \\
\text { Parent report: TV viewing negatively associated with TV rules }(B=0.18 ; \mathrm{SE}=0.08 ; \beta=-0.22 ; p<0.01) \text {. }\end{array}$ \\
\hline 6 & Springer et al. $(2010)^{32}$ & $\begin{array}{l}\text { Logistic regression: children with rules were } 1.68 \text { times more likely to view } \leq 2 \text { hours/day on weekdays } \\
(\mathrm{OR}=1.68 ; 95 \% \mathrm{Cl}=1.22,2.32 ; p<0.05) \text { and } \mathrm{I} .42 \text { more likely to view } \leq 2 \text { hours/day on weekends } \\
(\mathrm{OR}=\mathrm{I} .42 ; 95 \% \mathrm{Cl}=\mathrm{I} .04,1.94 ; p<0.05) \text {. }\end{array}$ \\
\hline 7 & $\begin{array}{l}\text { Van den Bulck et al. } \\
(2000)^{33}\end{array}$ & $\begin{array}{l}\text { Hierarchical stepwise regression: Mother's restrictive mediation negatively associated with girls TV } \\
\text { viewing }(\beta=-0.19 ; p<0.001)\end{array}$ \\
\hline 9 & Holman et al. $(1982)^{35}$ & $\begin{array}{l}\text { Correlations: Children with discriminating parents (able to specify unsuitable programs) likely to view } \\
\text { more commercial TV }(r=0.15 ; p<0.0 \mathrm{I}) \text {. Child selection of their own TV programs was associated with } \\
\text { higher viewing }(r=0.30, p<0.00 \mathrm{I}) \text {. Parent selection of TV programs for the child was not associated } \\
\text { with higher TV viewing }(r=0.00 ; p>0.05) \text {. }\end{array}$ \\
\hline 10 & Sun $(2009)^{36}$ & $\begin{array}{l}\text { Correlations: Child TV viewing not significantly correlated with total limits and content restrictions } \\
\text { (restrictive mediation) for urban }(r=0.044 ; p>0.05) \text { or rural }(r=-0.002 ; p>0.05) \text { children. }\end{array}$ \\
\hline 11 & Barkin et al. $(2006)^{37}$ & $\begin{array}{l}\text { Media exposure time varied depending on media mediation style; restrictive style }(M=2.4 \text { hours/day; } \\
S D=I .3 ; p \text { n.r), instructive style }(M=2.9 \text { hours/day; } S D=1.5 ; p \text { n.r }) \text { and unlimited }(M=4.1 \text { hours/day; } \\
S D=I .6 ; p \text { n.r). }\end{array}$ \\
\hline 12 & Barradas et al. $(2007)^{38}$ & $\begin{array}{l}\text { Linear regression: Parent rules were associated with TV viewing at ages } 10-12(\beta=2.43 ; p<0.00 \mathrm{I}), \\
13-15(\beta=1.64 ; p<0.0 \mathrm{I}) \text {, and } 16-18(\beta=0.98 ; p>0.05) \text {. The associations persisted following adjustment } \\
\text { for parental variables: age I0-12 }(\beta=2.15 ; p<0.00 \mathrm{I}), 13-15(\beta=1.46 ; p<0.0 \mathrm{I}), 16-18(\beta=0.82 ; p>0.05) \\
\text { and after further adjustments for demographics: age } 10-12(\beta=2.15 ; p<0.00 \mathrm{I}), 13-15(\beta=1.73 ; p<0.0 \mathrm{I}) \text {, } \\
16-18(\beta=1.1 \mathrm{I} ; p<0.05) \text {. }\end{array}$ \\
\hline 14 & Carlson et al. $(2010)^{40}$ & $\begin{array}{l}\text { Logistic regression: Children who really agree that their parents have rules over total TV viewing time } \\
\text { are less likely to exceed } 2 \text { hours/day of } \mathrm{SV}(\mathrm{OR}=0.66 ; 95 \% \mathrm{Cl}=0.55,0.80 ; p \mathrm{nr}) \text { than those who really } \\
\text { disagree that their parents have rules. }\end{array}$ \\
\hline 16 & Hume et al. $(2010)^{42}$ & $\begin{array}{l}\text { Logistic regression: Parent TV viewing rules were negatively associated with likelihood of viewing } \\
>2 \text { hours/day (unadjusted } \mathrm{OR}=0.6 ; 95 \% \mathrm{Cl}=0.44,0.86 ; p \leq 0.0 \mathrm{I} \text { ). When model was adjusted for all } \\
\text { other significant variables and clustering by class, association was attenuated (adjusted } \mathrm{OR} \text { and } \mathrm{Cl} \text { not } \\
\text { reported). }\end{array}$ \\
\hline 17 & Jago et al $(20 \mathrm{II})^{43}$ & $\begin{array}{l}\text { Multinomial regression: RRR for children viewing } 2-4 \text { hours/day TV was } 2.2 \text { times higher for children } \\
\text { with low restriction of sedentary time compared to those with high restrictions (RRR: } 2.16 ; 95 \% \\
\mathrm{Cl}=\mathrm{I} .19,3.9 \mathrm{I} ; p=0.0 \mathrm{I} 0 \text { ). The risk of viewing }>4 \text { hours/day TV was } 3.27 \text { times higher for children } \\
\text { with low restrictions compared to those with high restrictions (RRR: } 3.27 ; 95 \% \mathrm{Cl}=1.28,8.34 ; p=0.013 \text { ). }\end{array}$ \\
\hline 20 & Te Velde et al. $(20 \mathrm{II})^{46}$ & $\begin{array}{l}\text { Generalized Estimating Equation Regression: Adjusted regression analyses: (log scale) Rules about } \\
\text { total TV viewing }(\beta=-0.126 ; 95 \% \mathrm{Cl}=-0.225,-0.026) \text { and times when child can view TV }(\beta=-0.193 \text {; } \\
95 \% \mathrm{Cl}=-0.294,-0.093) \text { were significantly related to TV viewing }(p<0.05 \text {, exact } p \text { not reported). }\end{array}$ \\
\hline
\end{tabular}




\section{Table 7. Summary of Results of Associations between Parenting Practices}

\section{and Screen-Viewing continued}

\begin{tabular}{|c|c|c|}
\hline ID & Study & Findings \\
\hline 21 & $\begin{array}{l}\text { Vandewater et al. } \\
(2005)^{47}\end{array}$ & $\begin{array}{l}\text { Correlations: TV time rules negatively associated with child viewing }(r=-0.14 ; p<0.00 \mathrm{I}) \text { and positively } \\
\text { associated with frequency of viewing TV }(r=0.17 ; p<0.00 \mathrm{I}) \text {. Program rules positively associated with } \\
\text { duration (minutes) }(r=0.10 ; p<0.00 \mathrm{I}) \text { and frequency of child TV viewing }(r=0.44 ; p<0.00 \mathrm{I}) \text {. } \\
\text { ANCOVA: Parents with time rules report that child viewed TV more frequently }(3.06) \text { than those with } \\
\text { no time rules }(2.78)(F=I 5.38 ; p ; \beta<0.00 \mathrm{I}) \text {. Parents with time rules report lower child viewing } \\
\text { (M=95.30 minutes/day) than those with none }(M=I 25.69 \text { minutes } / \text { day) }(F=16.62 ; p<0.00 \mathrm{I}) \text {. } \\
\text { ANCOVA: Parents with program rules report higher frequency of child TV viewing }(3 . \mathrm{I} 4) \text { than those } \\
\text { with no program rules }(2.07)(F=I 55.83 ; p<0.00 \mathrm{I}) \text {. Program rules associated with higher viewing times } \\
\text { for children }(M=I I I .95 \text { minutes/day) than those without such rules }(M=79.48)(F=10.9 \mathrm{I} ; p<0.00 \mathrm{I}) \text {. }\end{array}$ \\
\hline 22 & $\begin{array}{l}\text { Griffore and Phenice. } \\
(1996)^{48}\end{array}$ & $\begin{array}{l}\text { t-test: Children who lived in houses with no rules watched more weekday TV than those who had rules } \\
(5.1 \text { hours/day, } S D=3.4 \text { vs. } 4.7 \text { hours } / d, S D=3.3, t=2.54, p=0.01 \mathrm{I}) \text {. Associations were not significant } \\
\text { at weekends; children with no rules watched } 5.5 \text { hours/day TV }(S D=3.7) \text {, children with rules watched } \\
5.2 \text { hours/day }(S D=3.4),(t=1.56 ; p=0.119) \text {. }\end{array}$ \\
\hline 23 & Hardy et al. $(2006)^{49}$ & $\begin{array}{l}\text { Logistic regression: Parent rules and child perception of rules not significant predictors of viewing time. } \\
\text { Likelihood of child with rules watching } \geq 2 \text { hours/day } T V \text { (in comparison to children with no rules, } \\
\text { respectively): } \\
\text { Turn off TV if child watches too much }(\mathrm{OR}=1.5 ; 95 \% \mathrm{Cl}=0.9,2.5 ; p>0.05) \\
\text { Do not let child watch too much } \mathrm{TV}(\mathrm{OR}=1.1 ; 95 \% \mathrm{Cl}=0.6,1.8 ; p>0.05) \\
\text { Prohibits TV during meal times }(\mathrm{OR}=1.0 ; 95 \% \mathrm{Cl}=0.9,1.2 ; p>0.05) \\
\text { TV time restrictions (OR }=0.6 ; 95 \% \mathrm{Cl}=0.3, I .3 ; p>0.05) \text {. }\end{array}$ \\
\hline 24 & Salmon et al. $(2005)^{50}$ & $\begin{array}{l}\text { Logistic regression: Children who have rules prohibiting TV during mealtime less likely to watch } \\
\geq 2 \text { hours/day TV (Boys: adjusted } \mathrm{OR}=0.6 ; 95 \% \mathrm{Cl}=0.3,0.9 ; p<0.05 \text {. Girls: adjusted } \mathrm{OR}=0.6 ; 95 \% \\
\mathrm{Cl}=0.4,0.9 ; p<0.05) \text {. } \\
\text { For boys, likelihood of viewing } \geq 2 \text { hours/day TV was reduced when parents restrict TV viewing } \\
\text { (adjusted } \mathrm{OR}=0.7 ; 95 \% \mathrm{Cl}=0.3, \mathrm{I} .4 ; p>0.05) \text { or when child is not allowed to watch TV before doing } \\
\text { homework }(\mathrm{OR}=0.6 ; 95 \% \mathrm{Cl}=0.3, \mathrm{I} . \mathrm{I} ; p>0.05) \text {. }\end{array}$ \\
\hline 26 & Truglio et al. $(1996)^{52}$ & $\begin{array}{l}\text { Multiple linear regression: Regulation of TV viewing has significant negative relationship with child's } \\
\text { general audience TV shows (drama, action, variety) at time I }(3 / 5 \text { years })\left(\beta=-0.20 ; \mathrm{R}^{2}=0.26 ; t=-03.78 \text {; }\right. \\
p<0.00 \mathrm{I}) \text { and time } 2\left(5 / 7 \text { years) }\left(\beta=-0.18 ; \mathrm{R}^{2}=0.30 ; t=-03.39 ; p<0.00 \mathrm{I}\right) \text {. }\right. \\
\text { TV regulation has little impact on child entertainment viewing (primarily cartoons) at Time I }(\beta=-0.05 ; \\
\left.\mathrm{R}^{2}=0.17 ; t=-0.83 ; p>0.10\right) \text { but has significant negative relationship at time } 2\left(\beta=-0.18 ; \mathrm{R}^{2}=0.20 ;\right. \\
t=-03.08 ; p<0.0 \mathrm{I}) \text {. }\end{array}$ \\
\hline 27 & $\begin{array}{l}\text { van Zutphen et al. } \\
(2007)^{53}\end{array}$ & $\begin{array}{l}\text { ANOVA with follow-up trend analysis: The strictness (e.g., tightness) of rules was associated with } \\
\text { overall TV time. Children with very tight rules had mean TV time of } 63 \text { minutes on previous day } \\
(\mathrm{SD}=2.7) \text {. Those with not very tight rules }(M=100 \text { min; } S D=4.7) \text {, and those that have no rules }(M=90 \text {; } \\
S D=2.4) \text { (linear trend } p<0.00 I) \text { ( } t \text { value and } p \text { nr). In comparison to children with very tight rules } \\
\text { (reference category), children with quite tight rules reported } 21 \text { additional minutes }(95 \% \mathrm{Cl}=13,28) \text {, } \\
\text { those with not very tight rules reported } 31 \text { more minutes }(95 \% \mathrm{Cl}=20,42) \text {, and those with no rules } \\
\text { reported } 20 \text { more minutes of viewing time }(95 \% \mathrm{Cl}=\mathrm{I}, 28) \text {. }\end{array}$ \\
\hline
\end{tabular}

\section{TV and DVD/VCR: Co-viewing}

\begin{tabular}{|c|c|c|}
\hline 10 & Sun $(2009)^{36}$ & $\begin{array}{l}\text { Correlations: Child TV viewing was significantly correlated with co-viewing for urban areas }(r=0.198 \text {; } \\
p \leq 0.0 \text { I) but not rural areas }(r=0.027 ; p>0.05) \text {. }\end{array}$ \\
\hline 15 & Davison et al. $(2005)^{41}$ & $\begin{array}{l}\text { Spearman rank correlations: Family co-viewing was cross-sectionally associated with TV at age } 9 \\
(r=0.35 ; p=<0.00 \mathrm{I}) \text { and II }(r=0.22 ; p=<0.00 \mathrm{I}) \text {. } \\
\text { Little evidence that co-viewing at } 9 \text { was associated with TV viewing at age II }(r=0.13 ; p>0.05) \text {. } \\
\text { Change in co-viewing from age } 9 \text { to II positively associated with change in TV viewing from } 9 \text { to II } \\
(r=0.18 ; p>0.05) \text {. }\end{array}$ \\
\hline 23 & Hardy et al. $(2006)^{49}$ & $\begin{array}{l}\text { Logistic regression: Children who co-view with parents more than once per week are more likely to } \\
\text { view } \geq 2 \text { hours/day TV: } 71.2 \% \text { who co-view more than once/week have } \geq 2 \text { hours/day TV viewing } \\
(\mathrm{OR}=2.8 ; 95 \% \mathrm{Cl}=\mathrm{I} .6,4.7 ; p<0.00 \mathrm{I}) \text {. } \\
\text { Logistical regression: In best predictive model (of family home environment and adolescents spending } \\
\geq 2 \text { hours/day TV viewing), adolescents watching TV with parents } \geq \mathrm{I} / \text { week had were more likely to } \\
\text { watch } \geq 2 \text { hours/day TV }(\mathrm{OR}=2.3 ; 95 \% \mathrm{Cl}=1.3,4.1 ; p=0.004) \text {. }\end{array}$ \\
\hline 8 & Warren et al. $(2002)^{34}$ & Linear regression: co-viewing was associated with child TV viewing $(\beta=0.16 ; p<0.01)$. \\
\hline
\end{tabular}




\begin{tabular}{|c|c|c|}
\hline ID & Study & Findings \\
\hline \multicolumn{3}{|r|}{ TV and DVD / VCR: Limit setting } \\
\hline 13 & Bauer et al. $(201 \mathrm{I})^{39}$ & $\begin{array}{l}\text { Hierarchical linear regression: Little evidence of parent encouragement for daughter to decrease TV } \\
\text { viewing (familial support for limiting TV use) being associated with TV (unadjusted standardized } \\
\beta=-0.14 ; p=0.446 \text {. Adjusted standardized } \beta=0.04 ; p=0.836 \text { ). }\end{array}$ \\
\hline 14 & Carlson et al. $(2010)^{40}$ & $\begin{array}{l}\text { Logistic regression: (parent report) Children who always/very often have limits are less likely to exceed } \\
2 \text { hours/day of } \mathrm{SV}(\mathrm{OR}=0.75 ; 95 \% \mathrm{Cl}=0.62,0.9 \mathrm{I} ; \mathrm{p} \mathrm{nr}) \text { than those who rarely/never have limits. Those } \\
\text { who sometimes have limits are also less likely to exceed } 2 \text { hours/day } \mathrm{SV}(\mathrm{OR}=0.95 ; 95 \% \mathrm{Cl}=0.77 \text {, } \\
\text { I.I7; } \mathrm{p} \text { n.r) than those who rarely/never have limits. }\end{array}$ \\
\hline 15 & Davison et al. $(2005)^{41}$ & $\begin{array}{l}\text { Spearman correlation: Girls TV restriction at age } 9 \text { positively associated with TV viewing at age II } \\
(r=0.18 ; p=<0.01) \text {. }\end{array}$ \\
\hline 18 & Lee et al. $(2009)^{44}$ & $\begin{array}{l}\text { Correlation: Parent limits correlated with TV time, at wave I (age } 0-12) r=-0.21 ; p<0.0 \mathrm{I} \text {, and wave } 2 \\
\text { (age 5-18) } r=-0.1 \mathrm{I} ; p \leq 0.05 \text {. } \\
\text { Linear regression parent limits negatively associated with TV time in cross-sectional analyses at } 0-4 \text { yrs } \\
(r=-0.16 ; p \leq 0.01), 5-8(r=-0.26 ; p \leq 0.01) \text { and } 9-12(r=-0.16 ; p \leq 0.0 I) \text {, associations attenuated in } \\
\text { longitudinal models } 0-4(r=-0.10 ; p>0.05), 5-8(r=0.00 ; p>0.05) \text {, and } 9-12(r=-0.05 ; p>.05) \text {. }\end{array}$ \\
\hline 25 & Wiecha et al. $(2001)^{5 I}$ & $\begin{array}{l}\text { Linear regression: Children with TV limits watched less TV than those with no limits }(3.0 \text { hours/day vs. } \\
3.8 \text { hours/day; } p<0.00 \mathrm{I}) \text {. Children whose parents set TV limits reported less TV show, video, and movie } \\
\text { viewing }(2.8 \text { hours/day vs. } 3.5 \text { hours/d; } p \text { nr). } \\
\text { Multivariate regression: No limits on TV viewing associated with increase of } 0.48 \text { hours viewing }(95 \% \\
\mathrm{Cl}=0.26,0.70 ; p<.0 \mathrm{I}), 0.42 \text { hour increase in TV shows, videos and movie viewing }(95 \% \mathrm{Cl}=0.21,0.63 \text {; } \\
p=<0.0 \mathrm{I}) \text {. }\end{array}$ \\
\hline
\end{tabular}

\section{TV and DVD / VCR: Supervision / Control}

\begin{tabular}{|c|c|c|}
\hline 4 & Patriarca et al. $(2009)^{30}$ & $\begin{array}{l}\text { Logistic regression: Parental control was not associated with likelihood of viewing }>2 \text { hours TV/day } \\
(\mathrm{OR}=0.93 ; 95 \% \mathrm{Cl}=0.68, \mathrm{I} .27 ; p=0.66) \text {. }\end{array}$ \\
\hline \multicolumn{3}{|r|}{ TV and DVD / VCR: Encouragement } \\
\hline 26 & Truglio et al. $(1996)^{52}$ & $\begin{array}{l}\text { Linear regression: Parental encouragement of TV viewing positively related to general audience viewing, } \\
\text { but only at Time } 2(5 \text { and } 7 \text { years old })(\beta=.20 ; t=3.54 ; p<0.00 \mathrm{I}) \text {. At Time I ( } 3 \text { and } 5 \text { years old) } \\
\beta=-0.06 ; t=-1.26 ; p>0.10 \text {. Relationship between parental encouragement and child entertainment } \\
\text { viewing at Time I is } \beta=-0.0 \mathrm{I}, t=-0.10, \mathrm{p}>0.10 \text {, at Time } 2 \beta=0.10, t=-1.65, \mathrm{p}<0.10 \text {. }\end{array}$ \\
\hline 27 & $\begin{array}{l}\text { van Zutphen et al. } \\
(2007)^{53}\end{array}$ & $\begin{array}{l}\text { ANOVA: TV time was higher when family environment enables easier access to TV, and for all indicators } \\
\text { of TV access, with dose-response association across categories }(p<0.001) \text {. F test values not reported. }\end{array}$ \\
\hline
\end{tabular}

\section{Computer: Rules}

\begin{tabular}{l|l|l}
\hline 2 & He et al. $(2010)^{28}$ & $\begin{array}{l}\text { Chi-squared: For homework: On weekdays, } 27.8 \% \text { of children with rules/limits use computer for } \\
<2 \text { hours/day }(p>0.05), \text { whereas } 30.4 \% \text { with rules/limits use for } \geq 2 \text { hours/day }(p>0.05) . \text { On weekends, } \\
\text { of those with rules/limits, } 22.7 \% \text { use for }<2 \text { hours/day and } 21.9 \% \text { for } \geq 2 \text { hours/day }(p>0.05) \\
\text { Non-homework: On weekdays, } 68.1 \% \text { of children with rules/limits use for }<2 \text { hours/day and } 61.0 \% \text { use } \\
\text { computer } \geq 2 \text { hours/day }(p>0.05) . \text { On weekends } 64.6 \% \text { use for }<2 \text { hours/day and } 47.4 \% \geq 2 \text { hours/day } \\
(p<0.0 I) .\end{array}$ \\
\hline 5 & Ramirez et al. $(201 \mathrm{I})^{31}$ & $\begin{array}{l}\text { Linear regression: Child-reported computer use negatively associated with computer rules }(B=-0.23 ; \\
\mathrm{SE}=0.12 ; \beta=-0.18 ; p<0.05) . \\
\text { In parent report model little evidence that computer use was associated with computer rules }(B=0.14 ; \\
\mathrm{SE}=0.09 ; \beta=-0.14 ; p>0.10) .\end{array}$ \\
\hline 4 & $\begin{array}{l}\text { Patriarca et al. }(2009)^{30} \\
(\beta=1.46 ; t=0.32 ; p=0.75) .\end{array}$ \\
\hline
\end{tabular}

\section{Computer: Limits}

\begin{tabular}{|c|c|c|}
\hline 18 & Lee et al. $(2009)^{44}$ & $\begin{array}{l}\text { Correlation: Parent limits correlated with PC time at wave I }(0-12)(r=0.03 ; p>0.05) \text {, and wave } 2 \\
(5-18)(r=-0.07 ; p<0.05) \text {. } \\
\text { Linear regression: Parental limits negatively associated with computer time at } 9-12(r=-0.14 ; p \leq 0.05) \text {. } \\
\text { No other significant associations cross-sectionally at } 0-9 \text { years }(r=-0.03 ; p>0.05), 5-13 \text { years }(r=0.08 \text {; } \\
p>0.05) \text {, or }>9 \text { years }(r=0.00 ; p>0.05) \text {. No significant longitudinal associations at } 0-9 \text { years }(r=0.04 \text {; } \\
p>0.05) \text { or } 5-13 \text { years }(r=0.02 ; p>0.05) \text {. }\end{array}$ \\
\hline
\end{tabular}




\section{Table 7. Summary of Results of Associations between Parenting Practices} and Screen-Viewing continued

\begin{tabular}{|c|c|c|}
\hline ID & Study & Findings \\
\hline 25 & Wiecha et al. $(200 \mathrm{I})^{5 \mathrm{I}}$ & $\begin{array}{l}\text { Multivariate regression: Children who had no TV limit had higher computer time }(M=0.34 \text { minutes/day; } \\
S D=0.46) \text { compared with those with TV limits }(M=0.25 \text { minutes } / \text { day; } S D=0.32 ; p \text { nr). }\end{array}$ \\
\hline 29 & Shin and Huh. $(20 \mathrm{II})^{55}$ & $\begin{array}{l}\text { Hierarchical regression: Parent mediation not significantly associated to stopping game playing } \\
(\beta=-0.03, p>0.05) \text {. }\end{array}$ \\
\hline
\end{tabular}

\section{Videogames: Rules}

\begin{tabular}{|c|c|c|}
\hline 2 & He et al. $(2010)^{28}$ & $\begin{array}{l}\text { Chi-squared: Time spent on videogames is lower for those who have rules. } \\
\text { Weekdays: More children who report videogame rules reported }<2 \text { hours/day screen time } \\
(58.8 \% ; p>0.05) \text { than } \geq 2 \text { hours/day }(53.6 \% ; p>0.05) \text {. } \\
\text { Weekends: Fewer children who reported } \geq 2 \text { hours/day screen time report rules }(33.1 \%) \text { compared } \\
\text { to those who reported }<2 \text { hours/day }(51.8 \% ; p>0.05) \text {. }\end{array}$ \\
\hline 4 & Patriarca et al. $(2009)^{30}$ & $\begin{array}{l}\text { Linear regression: Parent control of playing videogames negatively associated with mean minutes of } \\
\text { videogame time per day }(\beta=-12.11 ; t=-2.21 ; p=0.028) \text {. }\end{array}$ \\
\hline 7 & $\begin{array}{l}\text { Van den Bulck and Van } \\
\text { den Bergh. }(2000)^{33}\end{array}$ & $\begin{array}{l}\text { Multiple regression: Mother's restrictive mediation of computer use negatively associated with girls' } \\
\text { computer game use }(\beta=-0.24 ; p<0.001) \text {. }\end{array}$ \\
\hline \multicolumn{3}{|r|}{ Video-games: Limits } \\
\hline 14 & Carlson et al. $(2010)^{40}$ & $\begin{array}{l}\text { Logistic regression (parent report): Children who do not have/play videogames were less likely to } \\
\text { exceed } 2 \text { hour/day SV }(\mathrm{OR}=0.87 ; 95 \% \mathrm{Cl}=0.67, \mathrm{I} . \mathrm{I} 3) \text { than those who rarely/never play videogames. } \\
\text { Children who always/very often have time limits on video-game use less likely to exceed } 2 \text { hours/day } \\
(\mathrm{OR}=0.73 ; 95 \% \mathrm{Cl}=0.73, \mathrm{I} . \mathrm{II}) \text { than those who rarely/never play videogames. Children who sometimes } \\
\text { have limits are more likely to exceed } 2 \text { hours/day than those who rarely/never have limits }(\mathrm{OR}=1.20 \text {; } \\
95 \% \mathrm{Cl}=0.96, \mathrm{I} .50) \text {. }\end{array}$ \\
\hline 18 & Lee et al. $(2009)^{44}$ & $\begin{array}{l}\text { Correlation: Parent limits associated with video game use time, wave I }(r=-0-.04 ; p>0.05) \text {, and wave } 2 \\
(r=-0.0 I ; p>0.05) \text {. } \\
\text { Parental limits negatively correlated with videogame time (at } 9-12 \text { to } I 4+)(r=-0.1 I ; p \leq .05) \text {. }\end{array}$ \\
\hline
\end{tabular}

\section{Video-games: Co-playing}

29 Shin and Huh. $(20 \mathrm{II})^{55} \quad$ Hierarchical regression: Parental mediation not significantly related to co-playing $(\beta=0.04, p>0.05)$ of video games.

ANOVA, analysis of variance; ANCOVA, analysis of covariance; SD, standard deviation; nr, not reported; OR, odds ratio; Cl, confidence interval; SV, screen-viewing.

focuses on changing parent behavior. Equally, if an intervention incorporates elements of parent-mediated changes to the child's behavior, such as helping the children to identify alternative activities in which to engage when presented with reductions in media opportunities, child reports of parenting practices may be useful. Thus, when considering the selection of measures or the creation of new measures, there is a need to be clear about how the media parenting information will be used.

\section{Implications for Future Development of SV Parenting Measures}

We identified only three studies that assessed aspects of the general parenting style in relation to SV ${ }^{43,45,54}$ Parenting styles are based on sound psychological literature and theoretical frameworks, and, as such, the measures used benefit from more clearly defined and separable constructs supported by empirical evidence. ${ }^{18}$ It is important to note, however, that only a very limited range of general parenting constructs have been examined in relation to SV. As such, there is a need to identify the key aspects of general parenting that might be important influences on youth SV.

This review has captured information on media parenting practices that have been assessed in the literature. The studies included in the review were conducted between 1982 and 2011, and SV behaviors and media parenting practices have changed significantly over this time period. For example, it was recently reported that many 10- to 11year-old children engage in multi-SV in which they are often using two or three SV devices, such as a computer, phone, and TV, at the same time. ${ }^{80}$ The parenting practices that a parent adopts in relation to these multiscreen viewing options is likely to be very different from those that might have been conducted 10 years ago, when viewing was largely confined to a fixed television or computer screen. Thus, in addition to developing more refined measures that can more reliably assess the media practices that were identified in this review, there is also a need to 


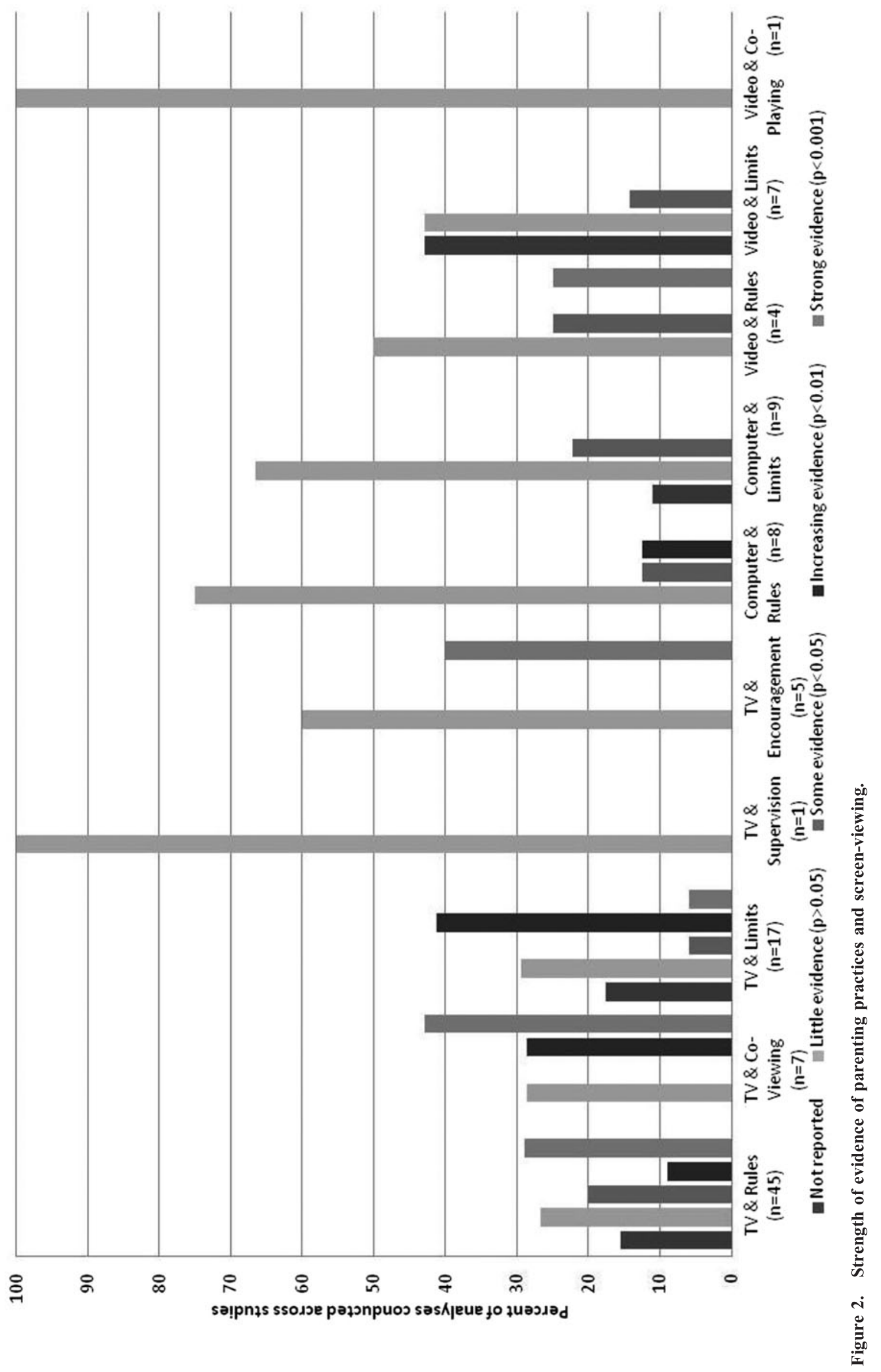


understand contemporary media parenting practices. Therefore, qualitative work with parents is required to examine these issues before developing future measures.

Current information on parenting practices and SV is limited by a lack of valid and/or reliable measures. It has been suggested by Aiken that the "the value of scientific data depends on the precision with which the variables under consideration are observed and measured." ${ }^{81}$ The data presented in this article would suggest that our current understanding of parental factors and SV is limited by the assessment methods. Many studies identified in the present review are limited by measures of exposure (i.e., parent styles or practices) and/or outcome (i.e., SV) that lack sufficient evidence for their reliability or validity.

Although a wide range of SV parenting practices have been examined in relation to youth SV, there is little consistency in the terms used to define SV parenting practices. For example, the term "rules" is used to cover a large range of different parenting practices (e.g., limits on time, limits on content or limits on time of day). The measurement of parenting practices is therefore at risk of the "Jingle-Jangle" fallacy ${ }^{82}$ in which two different constructs are labeled similarly (e.g., different/combinations of rules being generically termed "rules") and thus incorrectly used interchangeably (Jingle fallacy) or where two practices carry different labels (e.g., "limit setting"/ "restrictive mediation") but actually reflect the same construct (Jangle fallacy). Thus, there is a need for agreement on common terminology that is used to describe parenting practices. The tools that are used to assess these specific practices also need to be developed.

Overall, the information yielded by this systematic review indicates that the measurement of SV parenting practices would benefit from more systematic, extensive, and transparent scale development and validation research, with particular focus on construct definition. This process could begin by achieving consensus on the definitions for the seven rule and two nonrule-based parenting practices that have been identified in this review. Qualitative work should then be conducted with parents and children to identify any additional parenting practices that have not been previously assessed. At this stage, it is critical to be clear about whether the new scale is intended to be parent or child reported and the rationale for that decision. An initial item pool should then be developed before experts (e.g., academics, parents, and practitioners) are consulted to test the item content relevance ${ }^{83}$ and guide item refinement. Statistical approaches to scale validation (e.g., exploratory and confirmatory factor analysis) can then be employed before constructs are labeled. It is also important to note that as scale development is an ongoing process in which evidence for the validity and reliability of scale scores is amassed, ${ }^{84}$ researchers should continue to explore and report the psychometric properties of the scales they use. Therefore, we propose that researchers should consider the following four recommendations when considering the development of new SV parenting measures.
1. Researchers should define and agree on SV parenting practice terms.

2. Researchers should develop and validate new scales to measure parenting practices from first principles with independent work for child- and parent-reported measures.

3. Where parenting practice data are reported from scales for which psychometric support is weak or not available, researchers should justify their inclusion of the measure and include data pertaining to validity and reliability from their own data set.

4. Peer reviewers and editors should ensure that evidence for the psychometric properties of scales is not overlooked in manuscripts submitted for publication.

\section{Strengths and Limitations}

The major strength of this article is the systematic review of the literature. It is important to recognize, however, that the findings are limited by the quality of the studies included in the review. The review examined parent practices, parent styles, and parent mediation as separate items. However, we are aware that in certain circumstances parent styles could moderate the effect of parenting practices. This article did not explore such relationships and is thus unable to comment on any such associations. The searches were also only conducted in English and therefore it is possible that further evidence in non-English language journals, which could have added to the body of literature, has been missed in this review.

\section{Conclusions}

This systematic review has identified 29 studies that have examined parenting styles and practices in relation to youth SV. ${ }^{27-55}$ The studies include child-reported measures, parent-reported measures, and dual-reported measures. Data have been collected on children and adolescents, and a wide range of analytical methods have been used. The heterogeneous nature of the study designs prevents a formal synthesis of findings. However, the data indicate that a wide range of measures have been used to assess SV parenting practices, but in general there is a lack of information about the validity and reliability of the tools. No study has assessed parenting styles or practices in relation to multiscreen viewing. There is a need for new measures that assess a range of media parenting practices that are relevant to multiple forms of SV and have been shown to reliably assess the targeted practice in the participant group.

\section{Acknowledgments}

This report is research arising from a Career Development Fellowship (to Dr. Jago) supported by the National Institute for Health Research. The views expressed in this publication are those of the authors and not necessarily those of the National Health Service (NHS), the National 
Institute for Health Research, or the Department of Health. The preconference to the 2012 International Society for Behavioral Nutrition and Physical Activity (ISBNPA) annual meeting, "Parenting Measurement: Current Status and Consensus Reports" and resulting manuscripts were made possible due to funding from the United States Department of Agriculture/Agricultural Research Service (USDA/ARS 2012-68001-19285) and the National Heart, Lung, and Blood Institute of the National Institutes of Health (R13HL114262).

\section{Author Disclosure Statement}

We have no competing interests to declare.

\section{References}

1. Stamatakis E, Hamer M, Dunstan DW. Screen-based entertainment time, all-cause mortality, and cardiovascular events: Populationbased study with ongoing mortality and hospital events follow-up. J Am Coll Cardiol 2011;57:292-299.

2. Grontved A, Hu FB. Television viewing and risk of type 2 diabetes, cardiovascular disease, and all-cause mortality: A metaanalysis. JAMA 2011;305:2448-2455.

3. Jago R, Baranowski T, Baranowski JC, et al. BMI from 3-6 y of age is predicted by TV viewing and physical activity, not diet. Int $J$ Obes Relat Metab Disord 2005;29:557-564.

4. Padez C, Mourao I, Moreira P, et al. Prevalence and risk factors for overweight and obesity in Portuguese children. Acta Paediatr 2005;94:1550-1557.

5. Carvalhal MM, Padez MC, Moreira PA, et al. Overweight and obesity related to activities in Portuguese children, 7-9 years. Eur J Public Health 2007;17:42-46.

6. Marshall SJ, Biddle SJH, Gorley T, et al. Relationships between media use, body fatness and physical activity in children and youth: A meta-analysis. Int J Obes Relat Metab Disord 2004; 28:1238-1246.

7. Ekelund U, Brage S, Froberg K, et al. TV viewing and physical activity are independently associated with metabolic risk in children: The European Youth Heart Study. PLoS Med 2006;3:e488.

8. Page AS, Cooper AR, Griew P, et al. Children's screen viewing is related to psychological difficulties irrespective of physical activity. Pediatrics 2010;126:e1011-e1017.

9. Hamer M, Stamatakis E, Mishra G. Psychological distress, television viewing, and physical activity in children aged 4 to 12 years. Pediatrics 2009;123:1263-1268.

10. Biddle SJ, Pearson N, Ross GM, et al. Tracking of sedentary behaviours of young people: A systematic review. Prev Med 2010; 51:345-351.

11. American Academy of Pediatrics-Committee on Public Education. Children. Adolescents and Television. Pediatrics 2001;107:423426.

12. Sisson SB, Church TS, Martin CK, et al. Profiles of sedentary behavior in children and adolescents: The US National Health and Nutrition Examination Survey, 2001-2006. Int J Pediatr Obes 2009:1-7.

13. Tremblay MS, Leblanc AG, Janssen I, et al. Canadian sedentary behaviour guidelines for children and youth. Appl Physiol Nutr Metab 2011;36:59-64.
14. Department of Health. Change 4 Life. 2009 Available at www.nhs .uk/change4life/Pages/Default.aspx Last accessed June 27, 2013.

15. Baranowski T, Jago R. Understanding mechanisms of change in children's physical activity programs. Exerc Sport Sci Rev 2005; 33:163-168.

16. Jago R, Fox KR, Page AS, et al. Parent and child physical activity and sedentary time: Do active parents foster active children? BMC Public Health 2010;10:194.

17. Jago R, Stamatakis E, Gama A, et al. Parental and child screenviewing time and home media environment. Am J Prev Med 2012;43:150-158.

18. Baumrind D. Current patterns of parental authority. Devel Psychol Mono 1971;4:101-103.

19. Darling N, Steinberg L. Parenting style as a context: An integrative model. Psychol Bull 1993;113:487-496.

20. Kremers SPJ, Brug J, de Vries H, et al. Parenting style and adolescent fruit consumption. Appetite 2003;41:43-50.

21. Patrick H, Nicklas TA, Hughes SO, et al. The benefits of authoritative feeding style: Caregiver feeding styles and children's food consumption patterns. Appetite 2005;44:243-249.

22. Jago R, Davison KK, Brockman R, et al. Parenting styles, parenting practices, and physical activity in 10- to 11-year olds. Prev Med 2011;52:44-47.

23. Hennessy E, Hughes SO, Goldberg JP, et al. Parent-child interactions and objectively measured child physical activity: A crosssectional study. Int J Behav Nutr Phys Act 2010;7:71.

24. Gustafson SL, Rhodes RE. Parental correlates of physical activity in children and early adolescents. Sports Med 2006;36:79-97.

25. Pugliese J, Tinsley B. Parental socialization of child and adolescent physical activity: A meta-analysis. J Fam Psychol 2007;21:331-343.

26. Moher D, Liberati A, Tetzlaff J, et al. Preferred reporting items for systematic reviews and meta-analyses: The PRISMA statement. BMJ 2009;339:b2535.

27. Barr R, Danziger C, Hilliard ME, et al. Amount, content and context of infant media exposure: A parental questionnaire and diary analysis. Int J Early Years Educ 2010;18:107-122.

28. He M, Piche L, Beynon C, et al. Screen-related sedentary behaviors: Children's and parents' attitudes, motivations, and practices. J Nutr Educ Behav 2010;42:17-25.

29. Lin CA, Atkin DJ. Parental mediation and rulemaking for adolescent use of television and VCRs. Journal of Broadcasting \& Electronic Media 1989;33:53-67.

30. Patriarca A, Di Giuseppe G, Albano L, et al. Use of television, videogames, and computer among children and adolescents in Italy. BMC Public Health 2009;9:139.

31. Ramirez ER, Norman GJ, Rosenberg DE, et al. Adolescent screen time and rules to limit screen time in the home. J Adolesc Health 2011;48:379-385.

32. Springer AE, Kelder SH, Barroso CS, et al. Parental influences on television watching among children living on the Texas-Mexico border. Prev Med 2010;51:112-117.

33. Van den Bulck J, Van den Bergh B. The influence of perceived parental guidance patterns on children's media use: Gender differences and media displacement. Journal of Broadcasting \& Electronic Media 2000;44:329-348.

34. Warren R, Gerke P, Kelly M. Is there enough time on the clock? Parental involvement and mediation of children's television viewing. Journal of Broadcasting and Electronic Media 2002;46: 87-101.

35. Holman J, Braithwaite V. Parental lifestyles and children's television viewing. Australian J of Psychol 1982;34:375-382. 
36. Sun T. Parental mediation of children's TV viewing in China: An urban-rural comparison. Young Consumers 2009;10:188-198.

37. Barkin S, Ip E, Richardson I, et al. Parental media mediation styles for children aged 2 to 11 years. Arch Pediatr Adolesc Med 2006; 160:395-401.

38. Barradas DT, Fulton JE, Blanck HM, et al. Parental influences on youth television viewing. J Pediatr 2007;151:369-373, 373 e1-e4.

39. Bauer KW, Neumark-Sztainer D, Fulkerson JA, et al. Familial correlates of adolescent girls' physical activity, television use, dietary intake, weight, and body composition. Int J Behav Nutr Phys Act 2011;8:25.

40. Carlson SA, Fulton JE, Lee SM, et al. Influence of limit-setting and participation in physical activity on youth screen time. Pediatrics 2010;126:e89-e96.

41. Davison KK, Francis LA, Birch LL. Links between parents' and girls' television viewing behaviors: A longitudinal examination. J Pediatr 2005;147:436-442.

42. Hume C, van der Horst $\mathrm{K}$, Brug J, et al. Understanding the correlates of adolescents' TV viewing: A social ecological approach. Int $J$ Pediatr Obes 2010;5:161-168.

43. Jago R, Davison K, Thompson JL, et al. Parental sedentary restriction, maternal parenting style and TV viewing among 10-11 year olds. Pediatrics 2011;128:e572-e578.

44. Lee S-J, Bartolic S, Vandewater EA. Predicting children's media use in the USA: Differences in cross-sectional and longitudinal analysis. Br J Dev Psychol 2009;27:123-143.

45. Padilla-Walker LM, Coyne SM. "Turn that thing off!" parent and adolescent predictors of proactive media monitoring. $J$ Adolesc 2011;34:705-715.

46. Te Velde SJ, van der Horst K, Oenema A, et al. Parental and home influences on adolescents' TV viewing: A mediation analysis. Int $J$ Pediatr Obes 2011;6:e364-e372.

47. Vandewater EA, Park S, Huang X, et al. No-You can't watch that: Parental rules and young children's media use. Am Behav Scientist 2005;48:608-623.

48. Griffore R, Phenice L. Rules and television viewing. Psycholog Rep 1996;78:814.

49. Hardy LL, Baur LA, Garnett SP, et al. Family and home correlates of television viewing in 12-13 year old adolescents: The Nepean Study. Int J Behav Nutr Phys Act 2006;3:24.

50. Salmon J, Timperio A, Telford A, et al. Association of family environment with children's television viewing and with low level of physical activity. Obes Res 2005;13:1939-1951.

51. Wiecha J, Sobol A, Peterson K, et al. Household television access: Associations with screen time, reading, and homework among youth. Ambulatory Pediatr 2001;1:244-251.

52. Truglio R, Murphy K, Oppenheimer S, et al. Predictors of children's entertainment television viewing: Why are they tuning in? $J$ Appl Dev Psychol 1996;17:475-493.

53. van Zutphen M, Bell A, Kremer P, et al. Association between the family environment and television viewing in Australian children. $J$ Paediatr Child Health 2007;43:458-463.

54. Gable S, Lutz S. Household, parent, and child contributions to childhood obesity. Family Relations 2000;49:293-300.

55. Shin W, Huh J. Parental mediation of teenagers' video game playing: Antecedents and consequences. New Media and Society 2011;13:945-962.

56. Schludermann E, Schludermann S. Replicability of factors in children's report of parent behavior (CRPBI). J Psychol 1970; 76:239-249.
57. Schludermann S, Schluderman E. Questionnaire for children and youth (CRPBI-30). University of Manitoba: Winnipeg, Manitoba, Canada, 1988.

58. Block JH. The Child-Rearing Practices Report (CRPR): A Set of $Q$ Items for Description of Parental Socialization Attitudes and Values. University of California, Berkeley: Berkeley, CA, 1965. Online document at http://faculty.tru.ca/wlroberts/block,1965.pdf Last accessed June 27, 2013.

59. Hoelscher DM, Day RS, Lee ES, et al. Measuring the prevalence of overweight in Texas schoolchildren. Am J Public Health 2004;94:1002-1008.

60. Windle M, Grunbaum JA, Elliott M, et al. Healthy passages. A multilevel, multimethod longitudinal study of adolescent health. Am J Prev Med 2004;27:164-172.

61. Davison KK, Li K, Baskin ML, et al. Measuring parental support for children's physical activity in white and African American parents: The Activity Support Scale for Multiple Groups (ACTSMG). Prev Med 2011;52:39-43.

62. Rideout V, Roberts DF, Foehr MA. Generation M: Media in the Lives of 8-18 Year Olds. Henry J. Kaiser Family Foundation: Menlo Park, CA, 2005.

63. Gortmaker SL, Peterson K, Wiecha J, et al. Reducing obesity via a school-based interdisciplinary intervention among youth. Arch Pediatr Adolesc Med 1999;153:409-418.

64. Norman GJ, Schmid BA, Sallis JF, et al. Psychosocial and environmental correlates of adolescent sedentary behaviors. Pediatrics 2005;116:908-916.

65. Robinson TN. Reducing children's television viewing to prevent obesity: A randomized controlled trial. JAMA 1999;282:15611567.

66. Patrick K, Calfas KJ, Norman GJ, et al. Randomized controlled trial of a primary care and home-based intervention for physical activity and nutrition behaviors: PACE + for adolescents. Arch Pediatr Adolesc Med 2006;160:128-136.

67. Wendel-Vos GC, Schuit AJ, Saris WH, et al. Reproducibility and relative validity of the short questionnaire to assess healthenhancing physical activity. J Clin Epidemiol 2003;56:11631169.

68. Verplanken B. Beyond frequency: Habit as mental construct. $B r J$ Soc Psychol 2006;45:639-656.

69. Anderson DR, Field DE, Collins PA, et al. Estimates of young children's time with television: A methodological comparison of parent reports with time-lapse video home observation. Child Dev 1985;56:1345-1357.

70. Anderson CA, Dill KE. Video games and aggressive thoughts, feelings, and behavior in the laboratory and in life. J Pers Soc Psychol 2000;78:772-790.

71. Chinapaw MJ, Slootmaker SM, Schuit AJ, et al. Reliability and validity of the Activity Questionnaire for Adults and Adolescents (AQuAA). BMC Med Res Methodol 2009;9:58.

72. Mendelsohn AL, Berkule SB, Tomopoulos S, et al. Infant television and video exposure associated with limited parent-child verbal interactions in low socioeconomic status households. Arch Pediatr Adolesc Med 2008;162:411-417.

73. McMurray RG, Ring KB, Treuth MS, et al. Comparison of two approaches to structured physical activity surveys for adolescents. Med Sci Sports Exerc 2004;36:2135-2143.

74. Dowda M, Pate RR, Felton GM, et al. Physical activities and sedentary pursuits in African American and Caucasian girls. Res $Q$ Exerc Sport 2004;75:352-360.

75. Valkenburg PM, Krcmar M, Peeters P, et al. Developing a scale to assess three styles of television mediation: "Instructive Mediation," 
"Restrictive Mediation," and "Social Coviewing". Journal of Broadcasting \& Electronic Media 1999;43:52-66.

76. Salmon J, Owen N, Crawford D, et al. Physical activity and sedentary behavior: A population-based study of barriers, enjoyment, and preference. Health Psychol 2003;22:178-188.

77. St. Peters M, Fitch M, Huston AC, et al. Television and families: What do young children watch with their parents? Child Dev 1991;62:1409-1423.

78. Robinson CC, Mandleco B, Olsen SF, et al. The Parenting Styles and Dimensions Questionnaire (PSQD). In: Perlmutter B, Touliatos J, Holden GW (eds), Handbook of Family Measurement Techniques. Sage: Thousand Oaks, 2001, pp. 319-321.

79. Sterne JA, Davey Smith G. Sifting the evidence-what's wrong with significance tests? BMJ 2001;322:226-231.

80. Jago R, Sebire SJ, Gorely T, et al. "I'm on it 24/7 at the moment": A qualitative examination of multi-screen viewing behaviours among UK 10-11 year olds. Int J Behav Nutr Phys Act 2011;8:85.

81. Aiken LR. Rating Scales and Checklists: Evaluating Behavior, Personality, and Attitudes. Wiley: New York, 1996.

82. Block J. A contrarian view of the five-factor approach to personality description. Psychol Bull 1995;117:187-215.
83. Dunn JGH, Bouffard M, Rogers T. Assessing item content-relevance in sport psychology scale-construction research: Issues and recommendations. Measurement in Physical Education and Exercise Science 1999;3:15-36.

84. Messick S. Validity of psychological assessment: Validation of inferences from persons' responses and performances as scientific inquiry into score meaning. Am Psychol 1995;50:741-749.

Address correspondence to: Russell Jago, PhD Professor of Pediatric Physical Activity and Public Health

Centre for Exercise, Nutrition, and Health Sciences, School for Policy Studies University of Bristol 8 Priory Road Bristol, United Kingdom BS8 1TZ

E-mail: Russ.Jago@bris.ac.uk 